\title{
Dimeric Tetracenomycin Derivatives from a Taklamakan Desert-derived Streptomyces sp. HDN154193
}

Li Xing, $\| \dagger$ Yimin Chang, $\| \dagger$ Xiaoqian Zhang, ${ }^{\dagger}$ Xuewen Hou, ${ }^{\dagger}$ Yaxin Han, ${ }^{\dagger}$ Mudassir

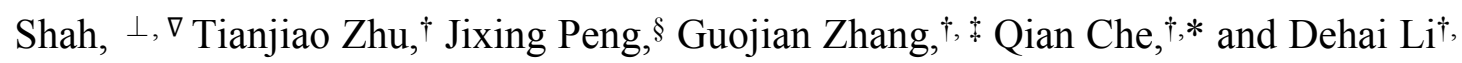
$\ddagger$

† School of Medicine and Pharmacy, Ocean University of China, Qingdao 266003, People's Republic of China

$¥$ Laboratory for Marine Drugs and Bioproducts of Qingdao National Laboratory for Marine Science and Technology, Qingdao, 266237, People's Republic of China

$\S$ Yellow Sea Fisheries Research Institute, Chinese Academy of Fishery Sciences, Qingdao 266071, People's Republic of China

$\perp$ Key Laboratory of Testing and Evaluation for Aquatic Product Safety and Quality, Ministry of Agriculture and Rural Affairs, People's Republic of China

$\nabla$ Department of Pharmacy, Abbottabad University of Science and Technology, Havellian, District Abbottabad, 22010, KPK, Pakistan 


\section{List of Supporting Information}

Figure S1. HPLC analysis of the crude extract of Streptomyces sp. HDN154193_...........4

Figure S2. The 16S rRNA sequences data of Streptomyces sp. HDN154193...................4

Figure S3. ${ }^{1} \mathrm{H}$ NMR (600 MHz, DMSO) spectrum of compound cis-1..........................5

Figure S4. ${ }^{13} \mathrm{C}$ NMR (150 MHz, DMSO) spectrum of compound cis-1........................5

Figure S5. HSQC spectrum of compound cis-1. .........................................................6

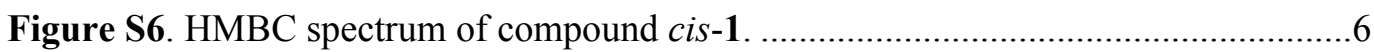

Figure S7. The HRESIMS spectrum of compound cis-1 .............................................

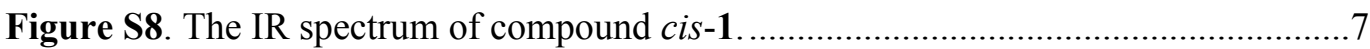

Figure S9. ${ }^{1} \mathrm{H}$ NMR (400 MHz, DMSO) spectrum of compound (+)-2 .......................... 8

Figure S10. ${ }^{13} \mathrm{C}$ NMR $(100 \mathrm{MHz}, \mathrm{DMSO})$ spectrum of compound $(+)-\mathbf{2} \ldots \ldots \ldots \ldots \ldots \ldots \ldots \ldots . . .8$

Figure S11. HSQC spectrum of compound (+)-2 ……..........................................

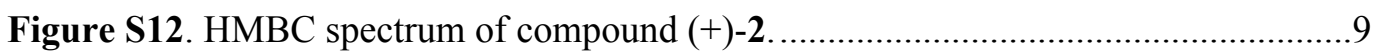

Figure S13. The HRESIMS spectrum of compound $(+)-2$. ...............................................

Figure S14. The IR spectrum of compound (+)-2 ....................................................10

Figure S15. ${ }^{1} \mathrm{H}$ NMR (500 MHz, DMSO) spectrum of compound (-)-2 .......................11

Figure S16. ${ }^{13} \mathrm{C}$ NMR (125 MHz, DMSO) spectrum of compound (-)-2 ......................11

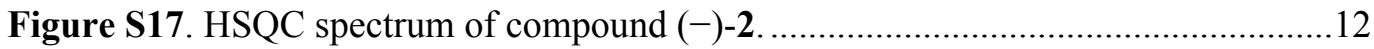

Figure S18. HMBC spectrum of compound (-)-2 _...................................................12

Figure S19. The HRESIMS spectrum of compound (-)-2 . .........................................13

Figure S20. The IR spectrum of compound (-)-2 ..................................................13

Figure S21. ${ }^{1} \mathrm{H}$ NMR (600 MHz, DMSO) spectrum of compound 3a by synthesis........14

Figure S22. ${ }^{13} \mathrm{C}$ NMR $(150 \mathrm{MHz}, \mathrm{DMSO})$ spectrum of compound 3a by synthesis. .....14

Figure S23. ${ }^{1} \mathrm{H}$ NMR (400 MHz, DMSO) spectrum of cis-1 by synthesis......................15

Figure S24. The HRESIMS spectrum of compound cis-1 by synthesis..........................15

Figure S25. ${ }^{1} \mathrm{H}$ NMR (400 MHz, DMSO) spectrum of (+)-2 by synthesis. ....................16

Figure S26. The HRESIMS spectrum of compound (+)-2 by synthesis. ........................16

Figure S27. ${ }^{1} \mathrm{H}$ NMR (500 MHz, DMSO) spectrum of compound (-)-2 by synthesis. ..17

Figure S28. The HRESIMS spectrum of compound (-)-2 by synthesis.

Table S1. Stack spectra and comparison of ${ }^{1} \mathrm{H}$ NMR for compounds 1, (+)-2 and (-)-2 18

Figure S29. DFT-optimized structures for low-energy conformers of $(+)-2 \ldots \ldots \ldots \ldots \ldots \ldots . . .18$

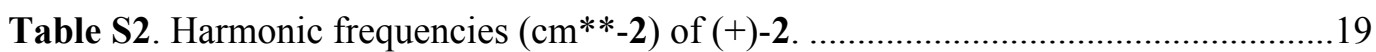


Table S3. Thermodynamic parametersand Boltzmann distributions of (+)-2 .19

Table S4. Cartesian coordinates of the low-energy reoptimized conformers of $(+)-2 \ldots .19$ 
Figure S1. HPLC analysis of the crude extract of Streptomyces sp. HDN154193.

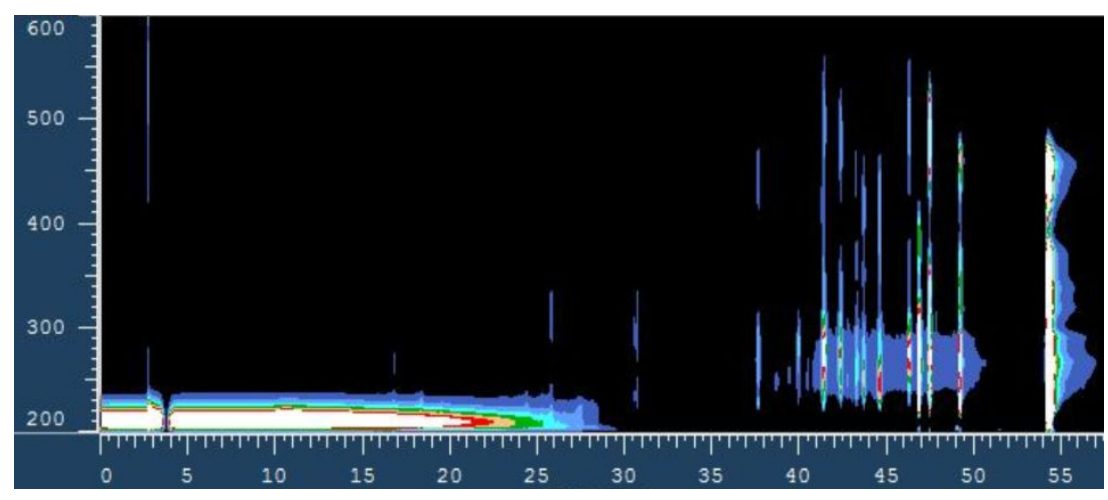

Figure S2. The 16S rRNA sequences data of Streptomyces sp. HDN154193.

GGTGGCCGGGGCGGCGTCTTACCATGCAGTCGAACGATGAACCTCCTTCGGGAGGGG ATTAGTGGCGAACGGGTGAGTAACACGTGGGCAATCTGCCCTGCACTCTGGGACAAG CCCTGGAAACGGGGTCTAATACCGGATACTGACCCGCTTGGGCATCCAAGCGGTTCG AAAGCTCCGGCGGTGCAGGATGAGCCCGCGGCCTATCAGCTTGTTGGTGAGGTAATG GCTCACCAAGGCGACGACGGGTAGCCGGCCTGAGAGGGCGACCGGCCACACTGGGA CTGAGACACGGCCCAGACTCCTACGGGAGGCAGCAGTGGGGAATATTGCACAATGGG CGAAAGCCTGATGCAGCGACGCCGCGTGAGGGATGACGGCCTTCGGGTTGTAAACCT CTTTCAGCAGGGAAGAAGCGAAAGTGACGGTACCTGCAGAAGAAGCGCCGGCTAAC TACGTGCCAGCAGCCGCGGTAATACGTAGGGCGCGAGCGTTGTCCGGAATTATTGGG CGTAAAGAGCTCGTAGGCGGCTTGTCACGTCGGTTGTGAAAGCCCGGGGCTTAACCC CGGGTCTGCAGTCGATACGGGCAGGCTAGAGTTCGGTAGGGGAGATCGGAATTCCTG GTGTAGCGGTGAAATGCGCAGATATCAGGAGGAACACCGGTGGCGAAGGCGGATCT CTGGGCCGATACTGACGCTGAGGAGCGAAAGCGTGGGGAGCGAACAGGATTAGATA CCCTGGTAGTCCACGCCGTAAACGGTGGGCACTAGGTGTGGGCGACATTCCACGTCG TCCGTGCCGCAGCTAACGCATTAAGTGCCCCGCCTGGGGAGTACGGCCGCAAGGCTA AAACTCAAAGGAATTGACGGGGGCCCGCACAAGCGGCGGAGCATGTGGCTTAATTCG ACGCAACGCGAAGAACCTTACCAAGGCTTGACATACACCGGAAAGCATCAGAGATG GTGCCCCCCTTGTGGTCGGTGTACAGGTGGTGCATGGCTGTCGTCAGCTCGTGTCGTG AGATGTTGGGTTAAGTCCCGCAACGAGCGCAACCCTTGTCCCGTGTTGCCAGCAGGC CCTTGTGGTGCTGGGGACTCACGGGAGACCGCCGGGGTCAACTCGGAGGAAGGTGGG GACGACGTCAAGTCATCATGCCCCTTATGTCTTGGGCTGCACACGTGCTACAATGGCC GGTACAATGAGCTGCGATACCGCGAGGTGGAGCGAATCTCAAAAAGCCGGTCTCAGT TCGGATTGGGGTCTGCAACTCGACCCCATGAAGTCGGAGTCGCTAGTAATCGCAGAT CAGCATTGCTGCGGTGAATACGTTCCCGGGCCTTGTACACACCGCCCGTCACGTCACG AAAGTCGGTAACACCCGAAGCCGGTGGCCCAACCCCTTGTGGGAGGGAGCTTCGAAG TGGACCGCCCT 
Figure S3. ${ }^{1} \mathrm{H}$ NMR (600 MHz, DMSO) spectrum of compound cis-1.

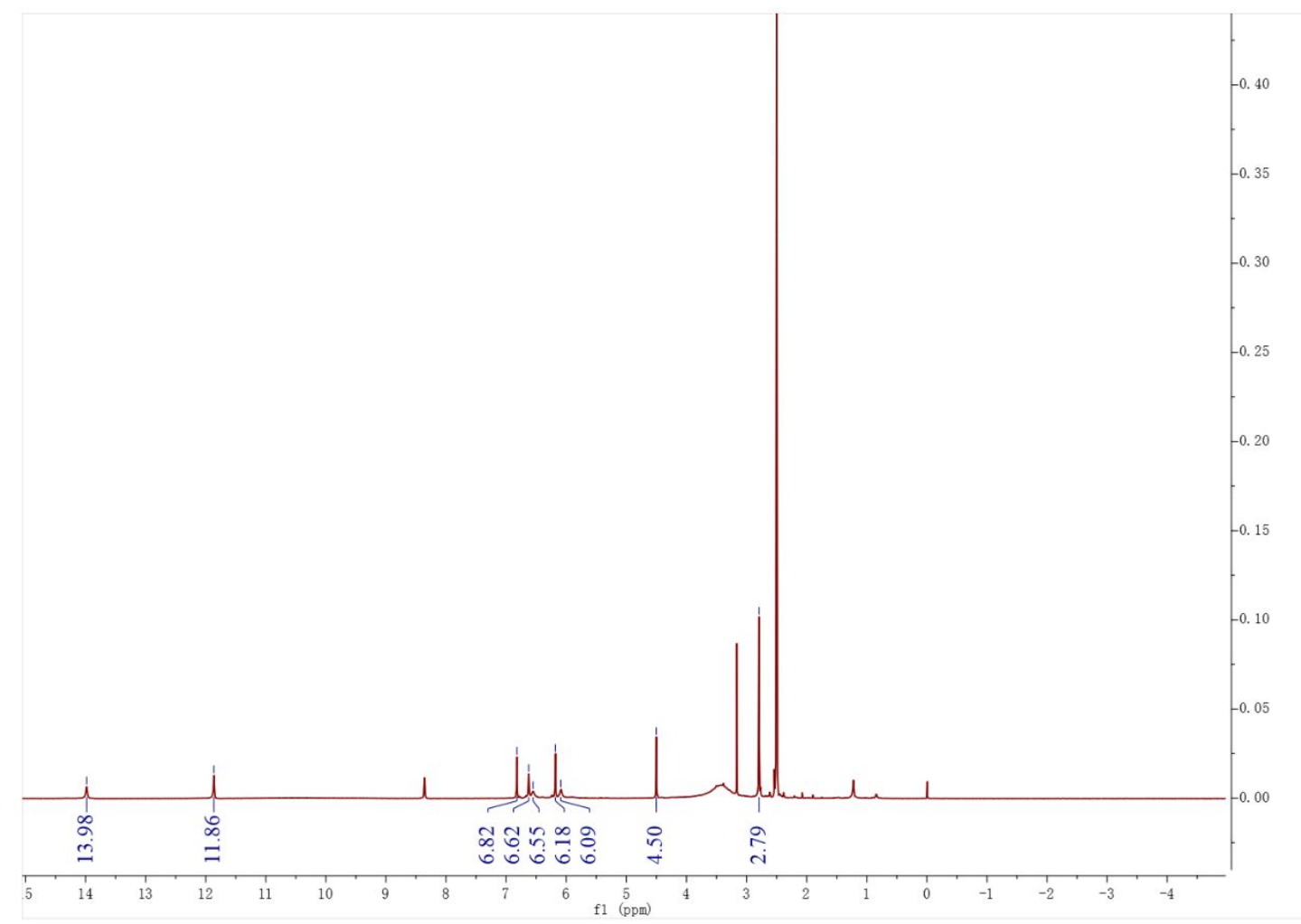

Figure S4. ${ }^{13} \mathrm{C}$ NMR (150 MHz, DMSO) spectrum of compound cis-1.

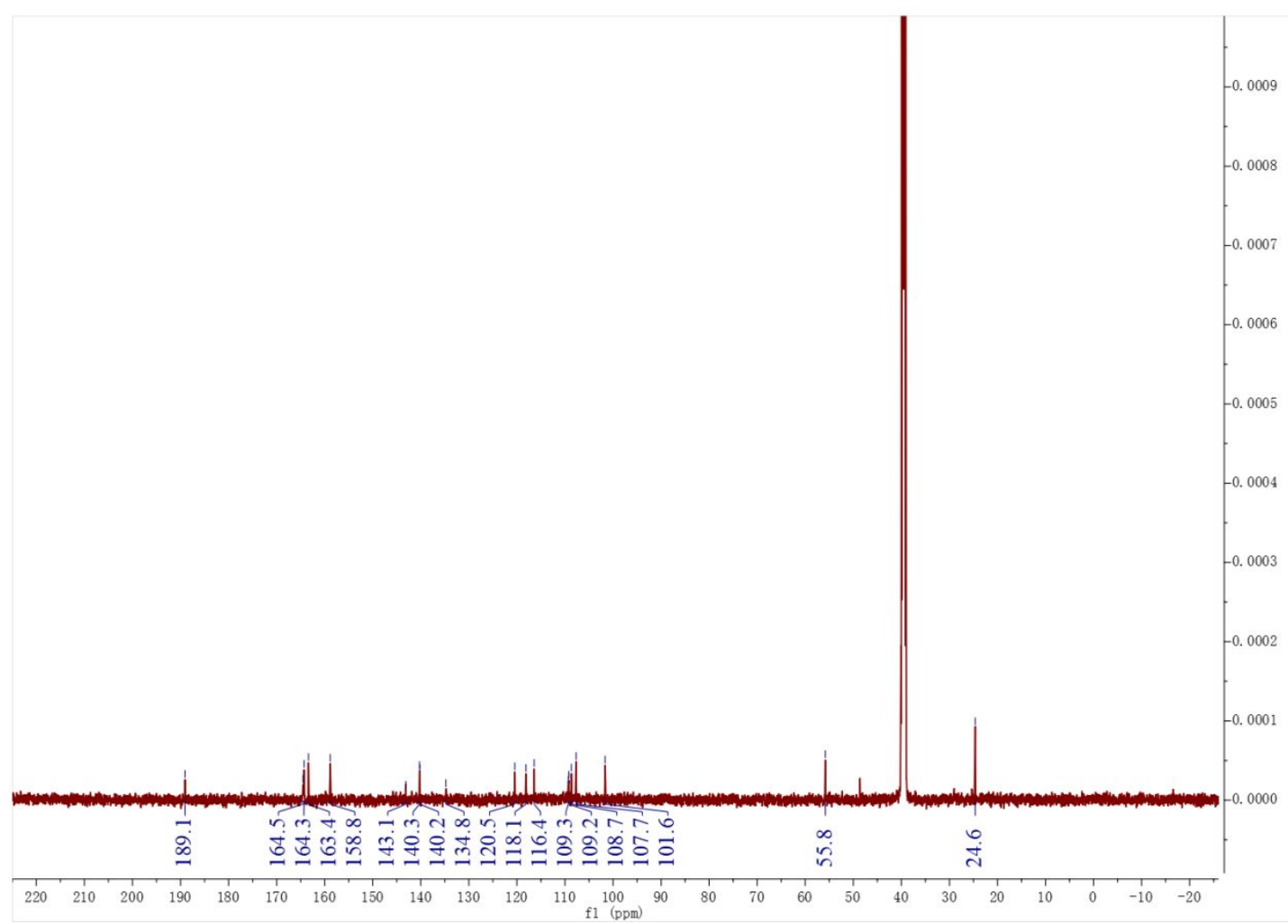


Figure S5. HSQC spectrum of compound cis-1.

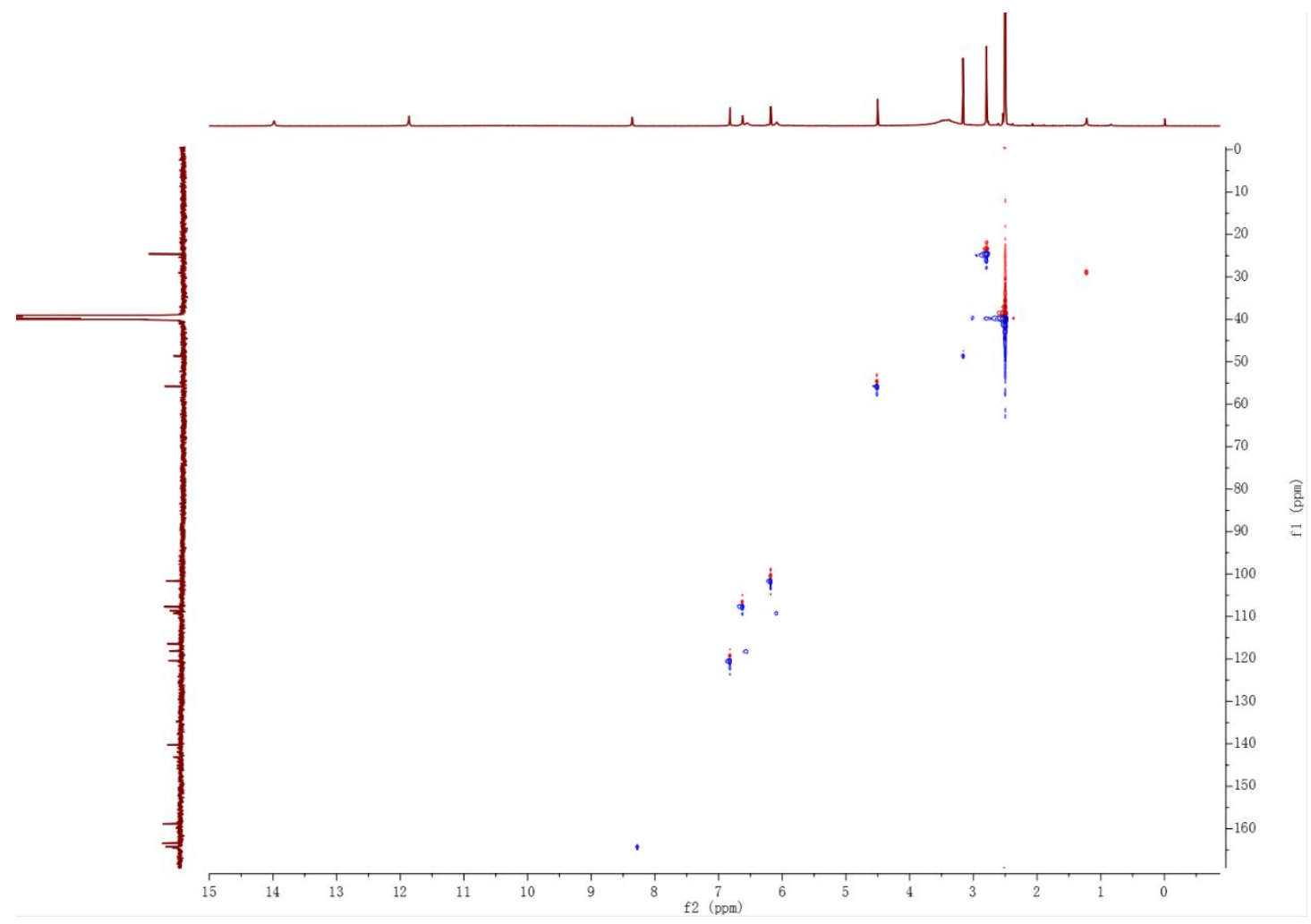

Figure S6. HMBC spectrum of compound cis-1.

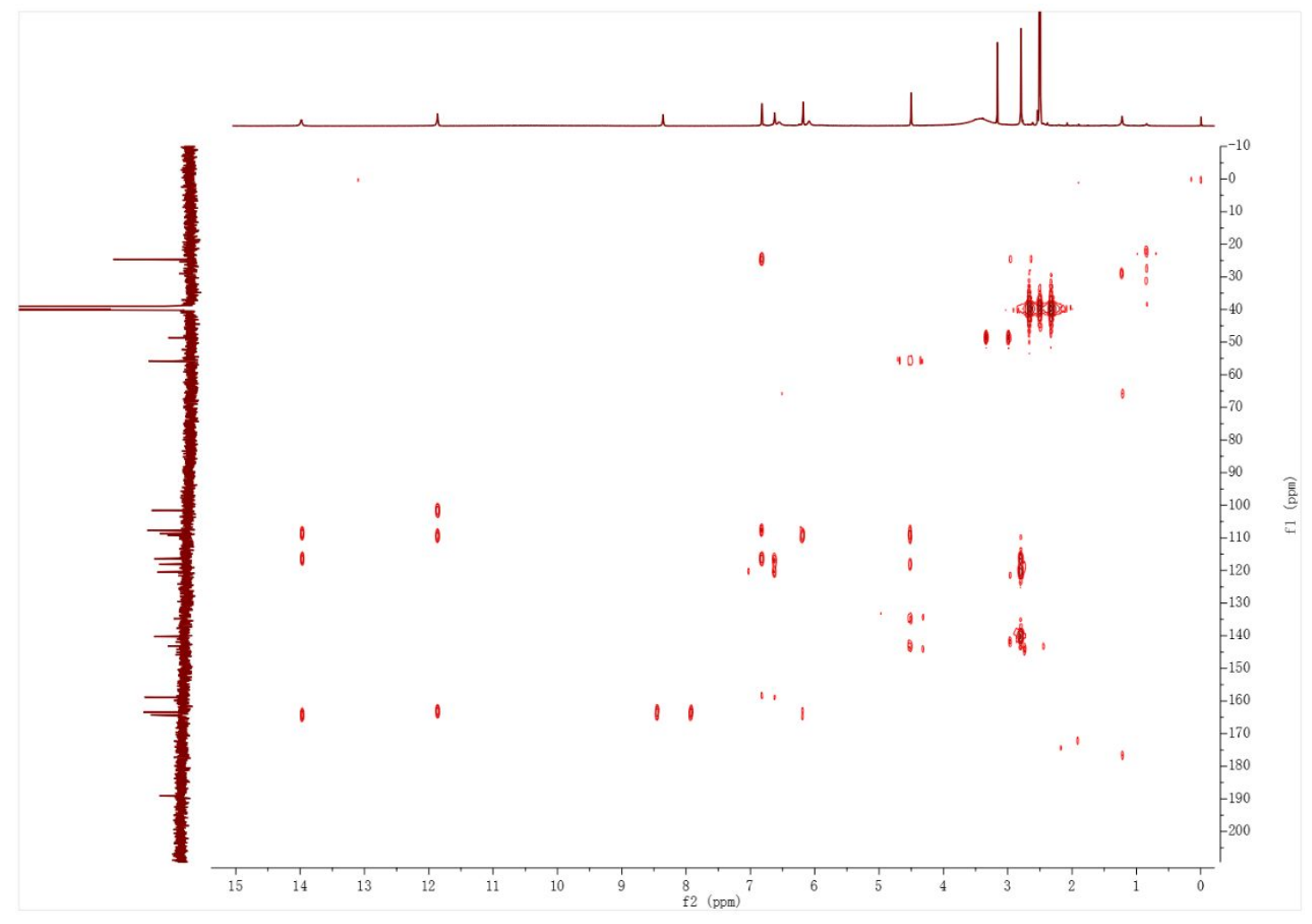


Figure S7. The HRESIMS spectrum of compound cis-1.

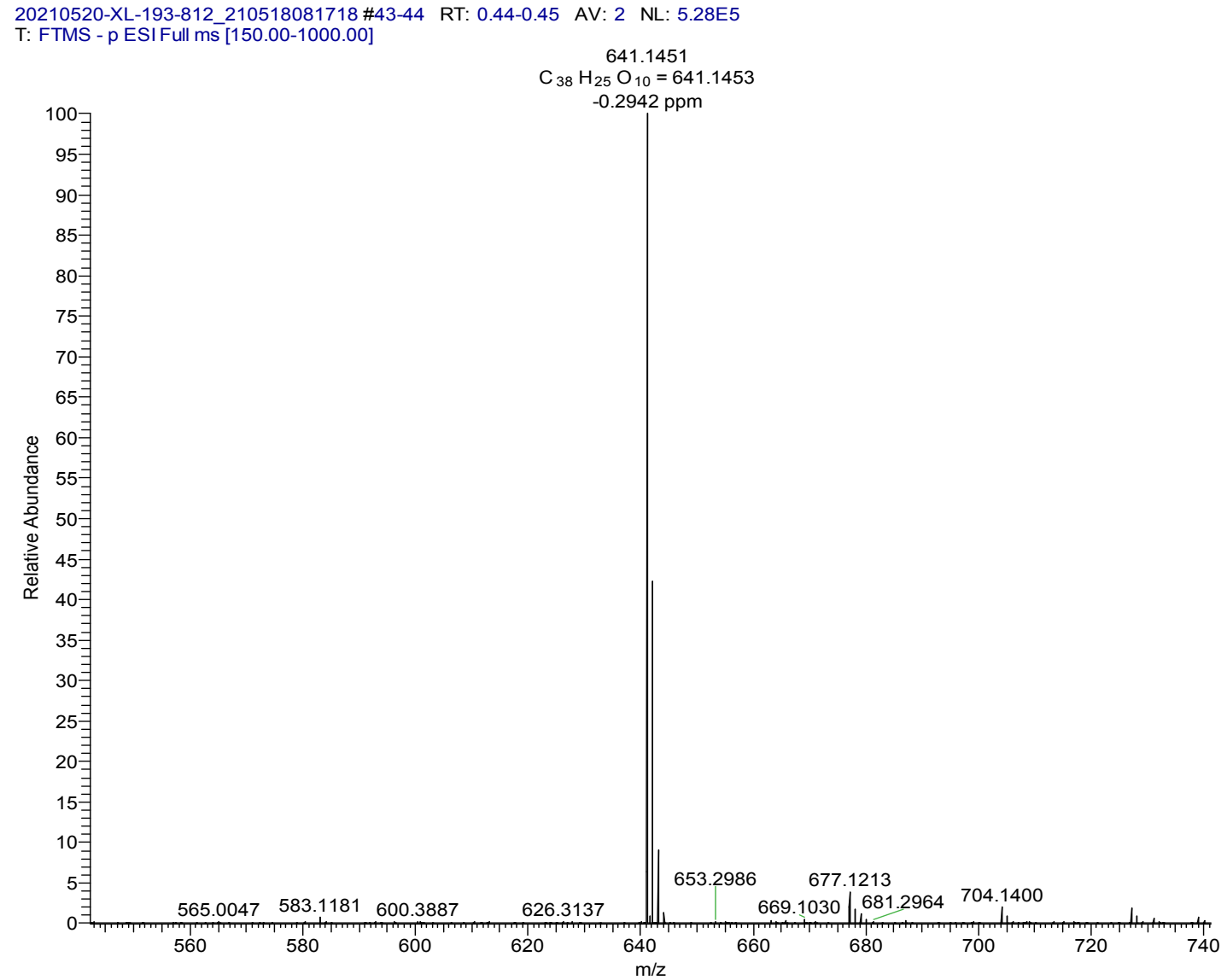

Figure S8. The IR spectrum of compound cis-1.

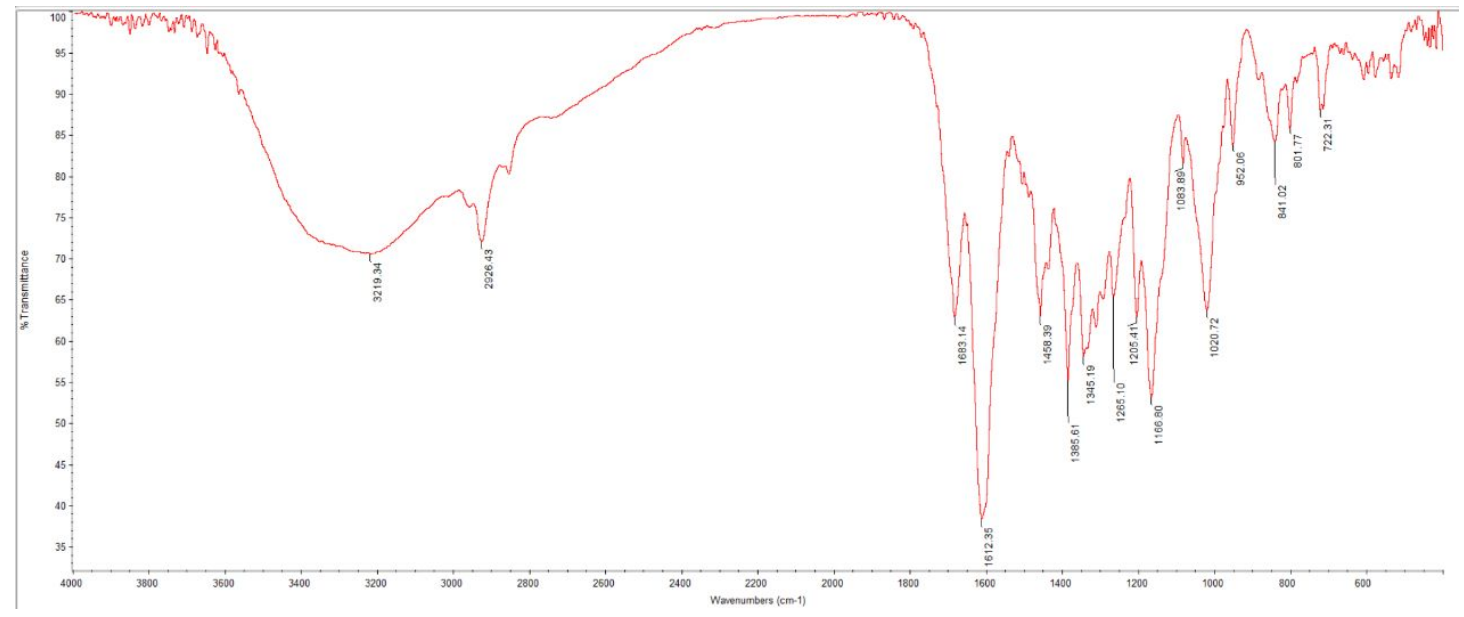


Figure S9. ${ }^{1} \mathrm{H}$ NMR (400 MHz, DMSO) spectrum of compound (+)-2.

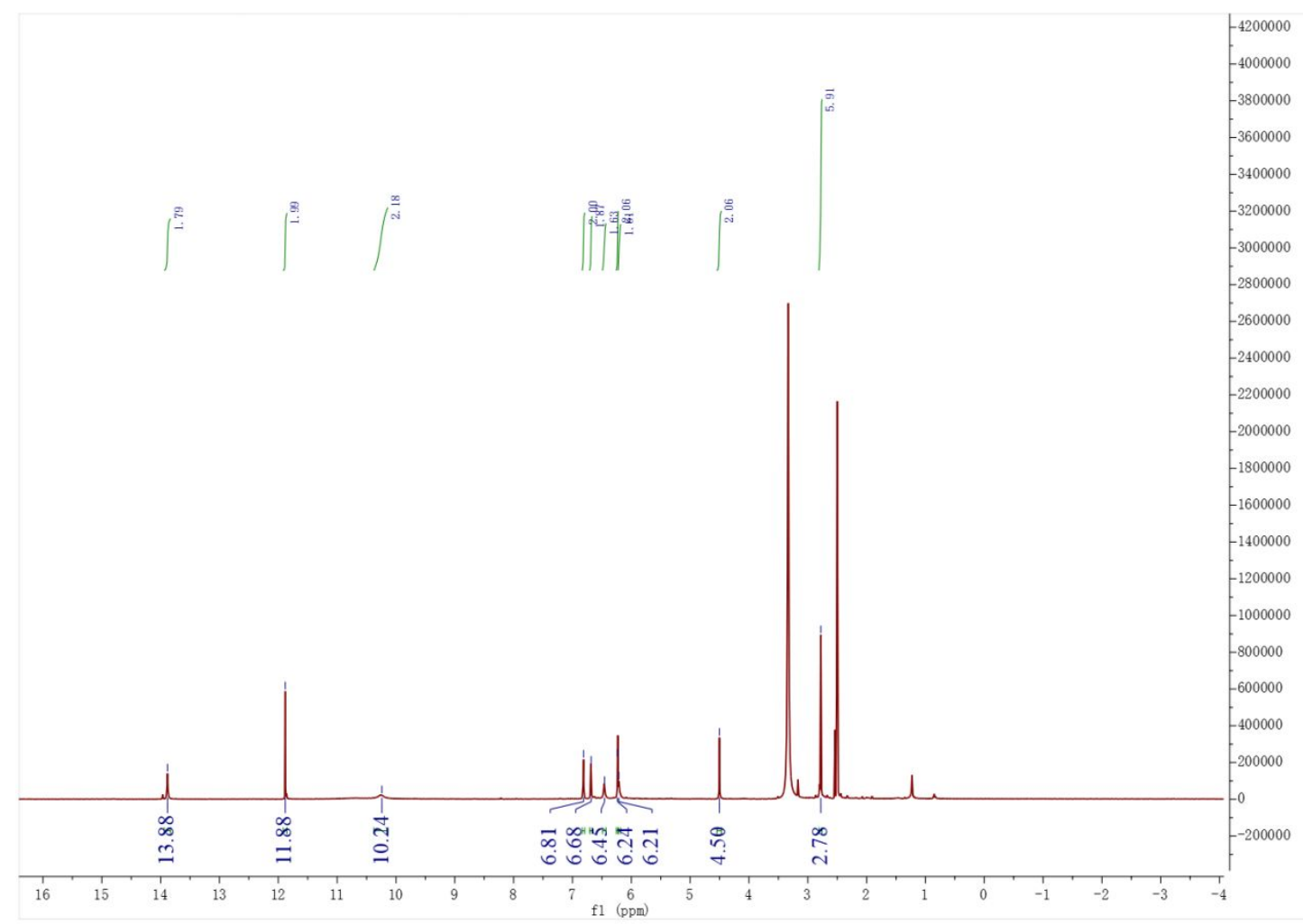

Figure S10. ${ }^{13} \mathrm{C}$ NMR (100 MHz, DMSO) spectrum of compound (+)-2.

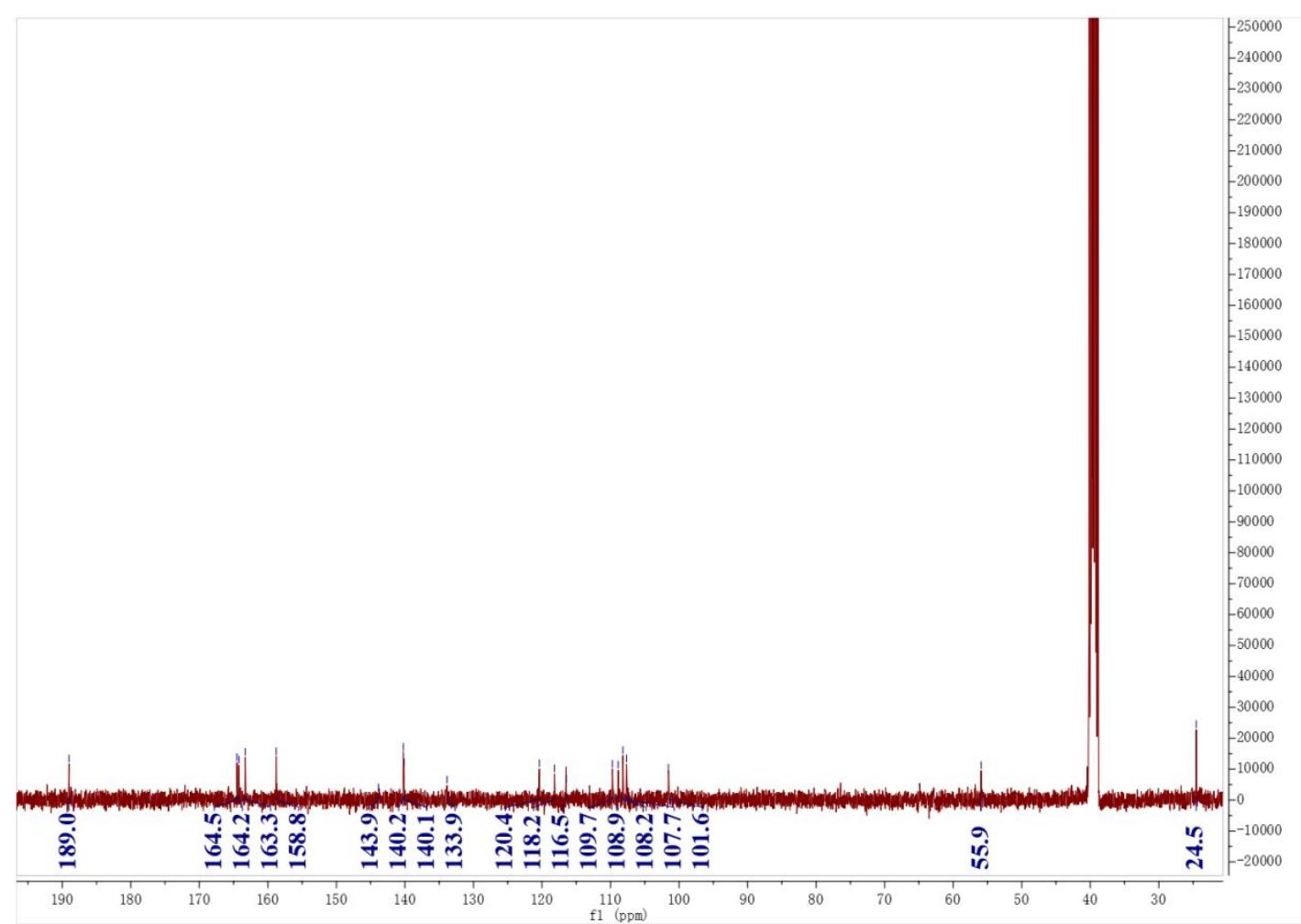


Figure S11. HSQC spectrum of compound (+)-2.

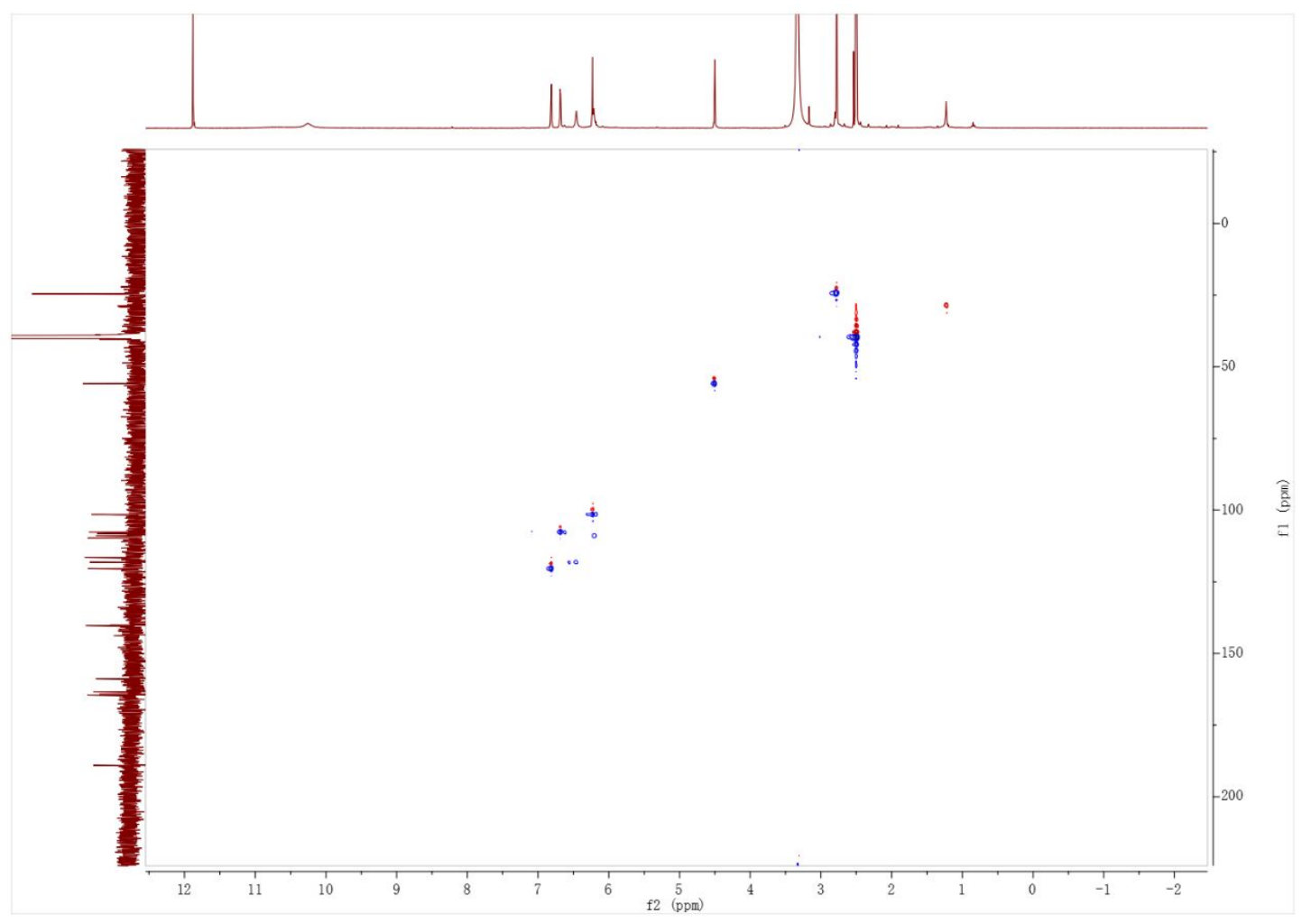

Figure S12. HMBC spectrum of compound (+)-2.

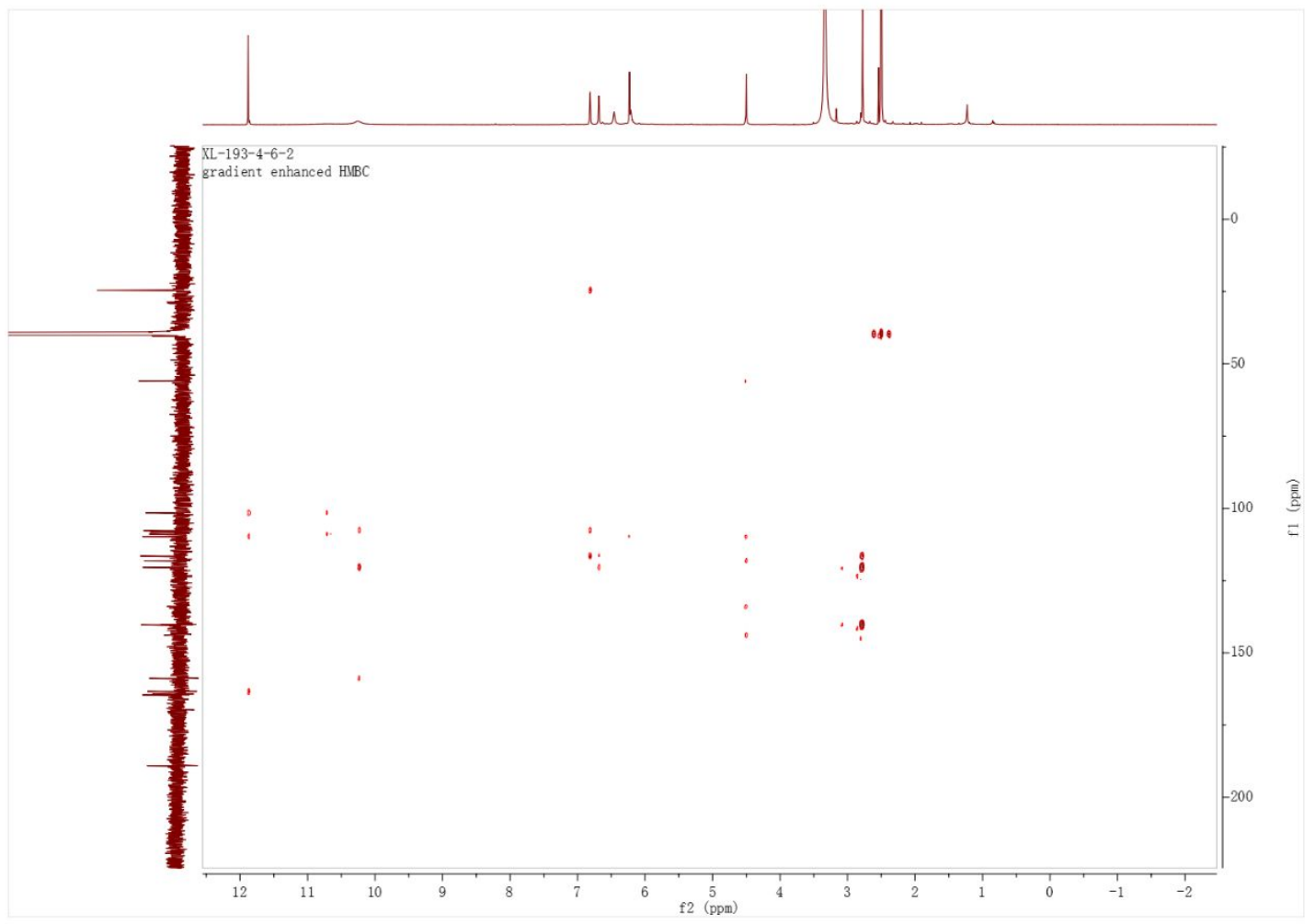

9 
Figure S13. The HRESIMS spectrum of compound (+)-2.

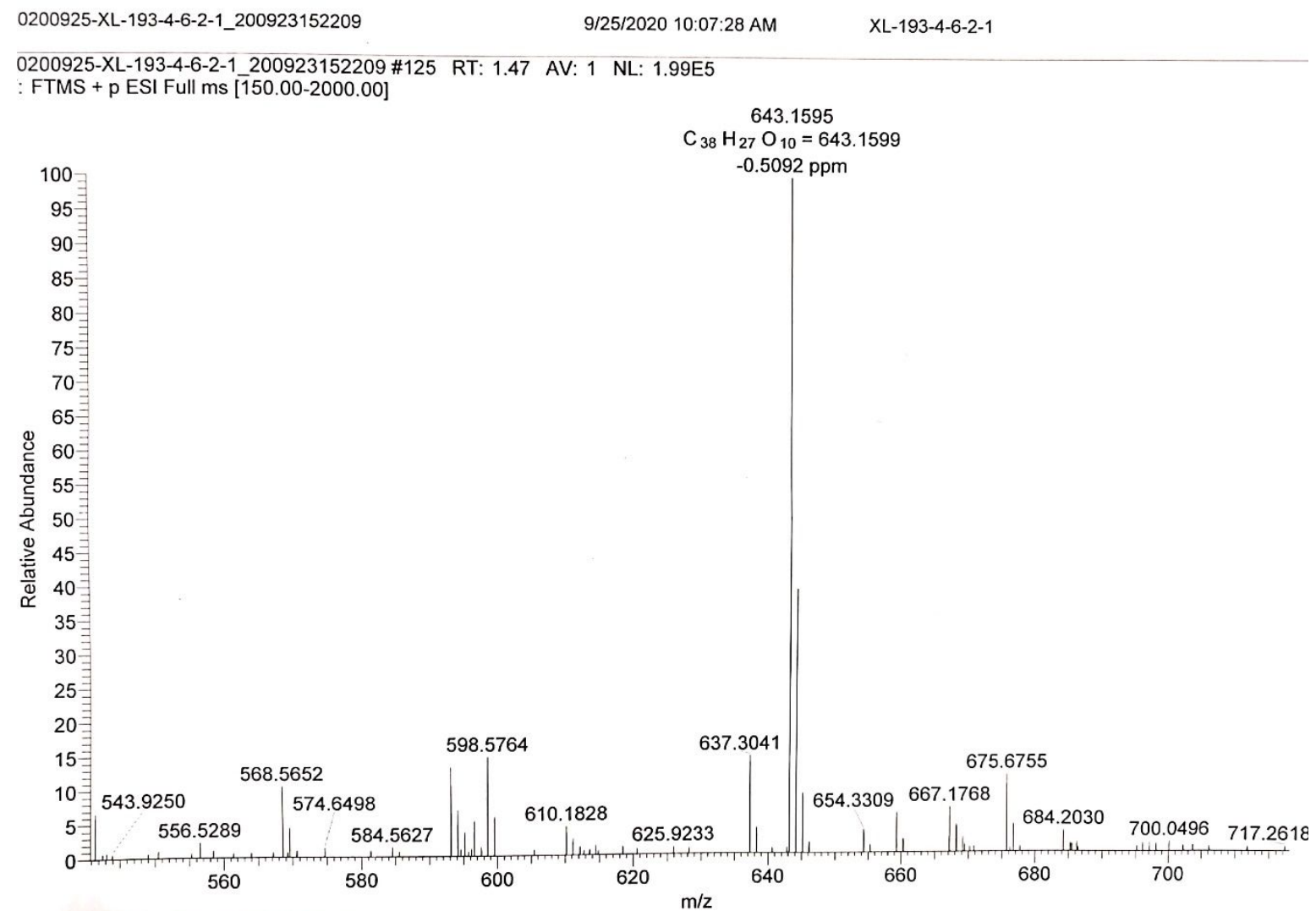

Figure S14. The IR spectrum of compound (+)-2.

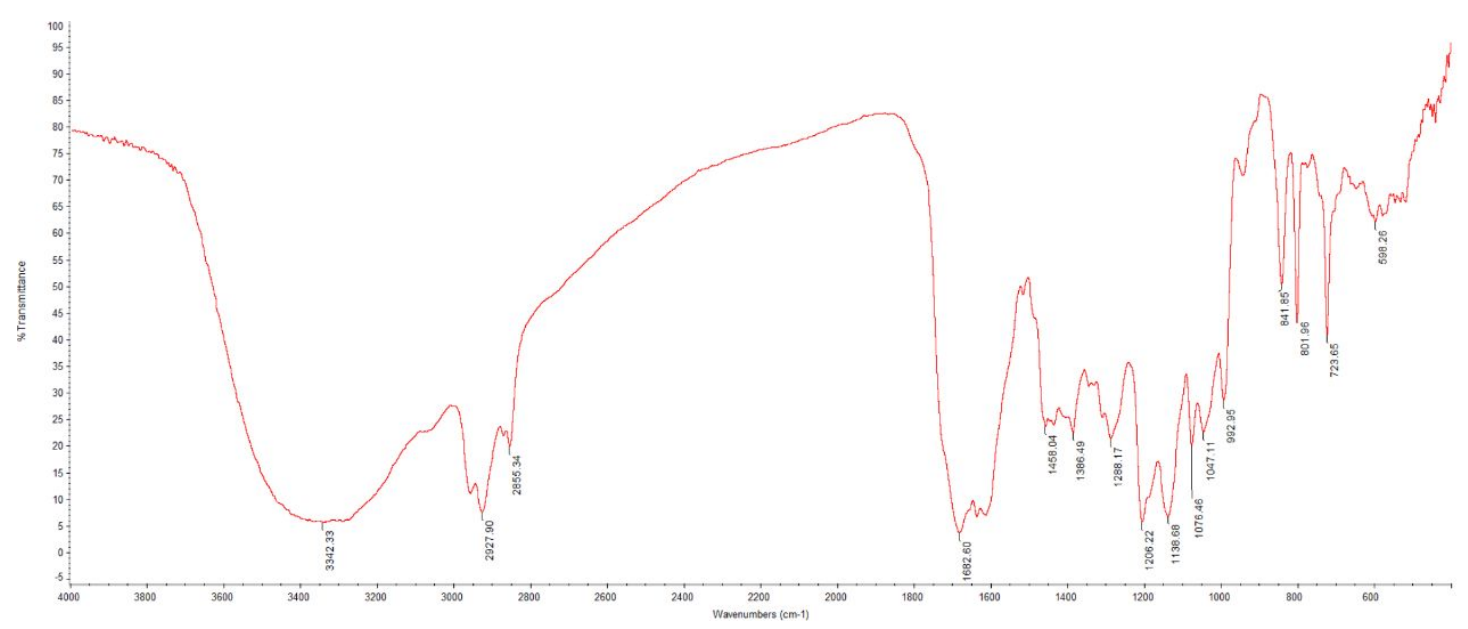


Figure S15. ${ }^{1} \mathrm{H}$ NMR (500 MHz, DMSO) spectrum of compound (-)-2.

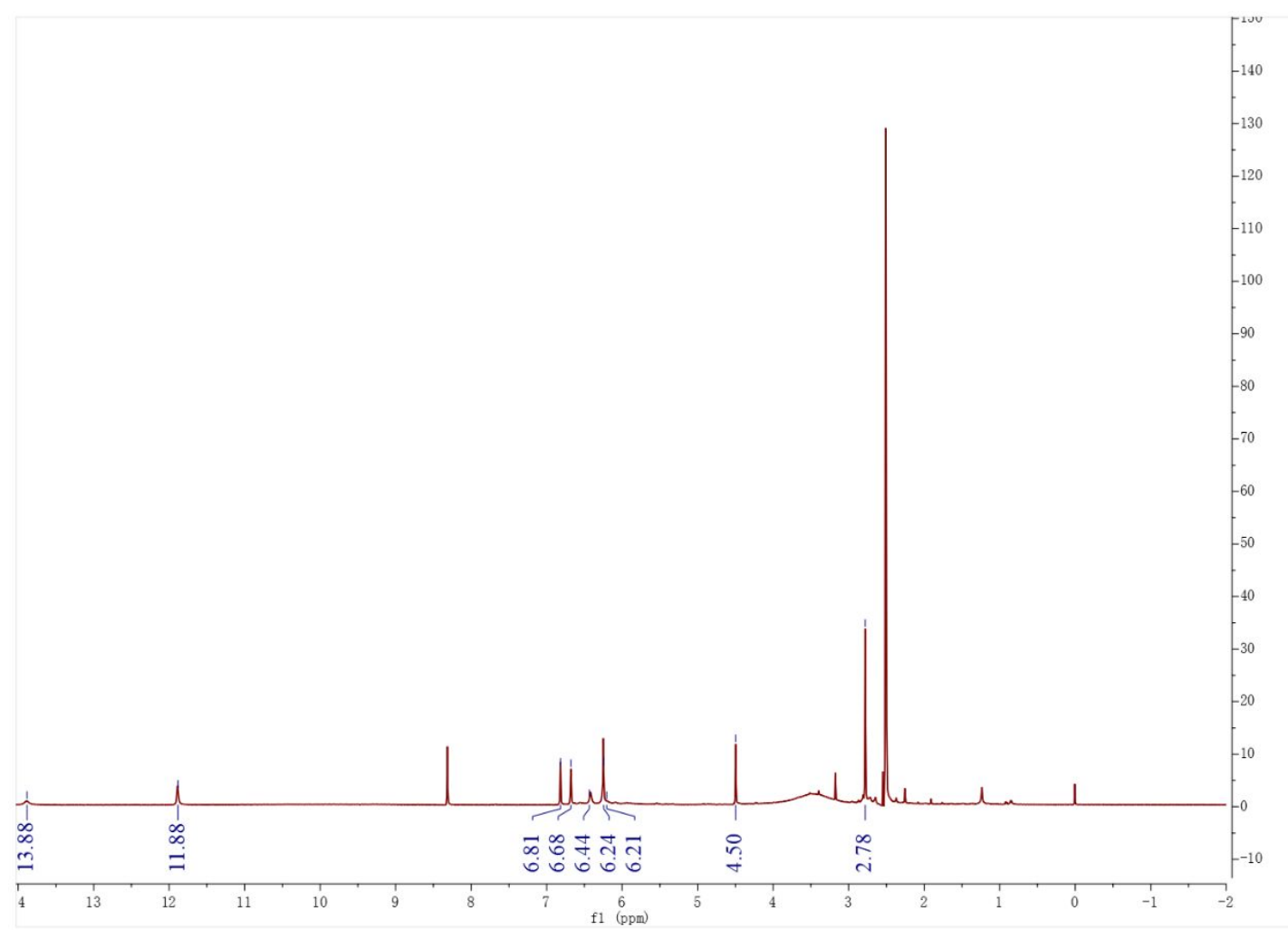

Figure S16. ${ }^{13} \mathrm{C}$ NMR (125 MHz, DMSO) spectrum of compound (-)-2.

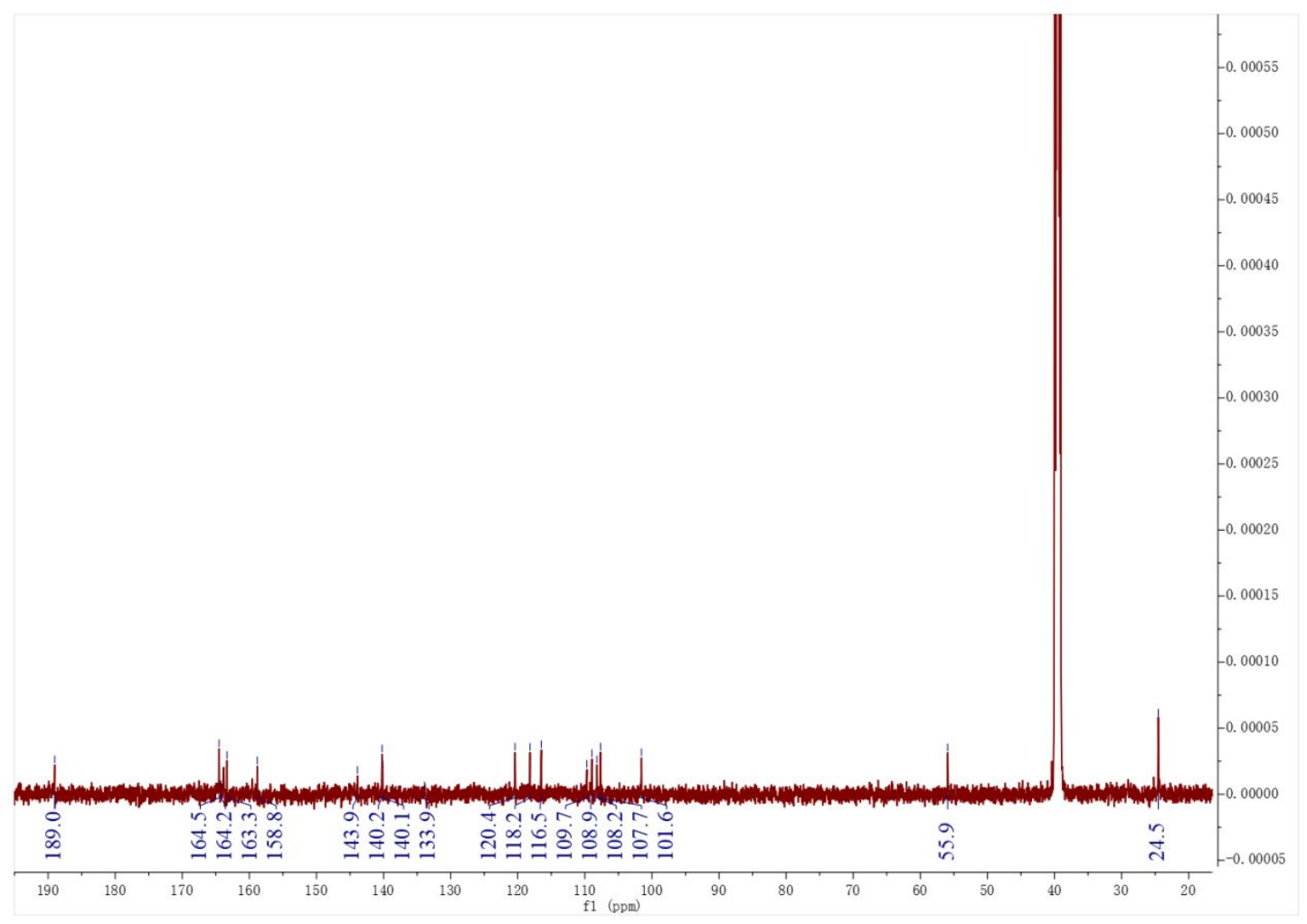




$$
\frac{T}{1}
$$


Figure S19. The HRESIMS spectrum of compound (-)-2.

20200925-XL-193-4-6-2-2_200923152209

9/25/2020 10:09:58 AM

$X L-193-4-6-2-2$

20200925-XL-193-4-6-2-2 200923152209 \#90-91 RT: 1.01-1.02 AV: 2 NL: 2.60E5

$\mathrm{T}:$ FTMS + p ESI Full ms [150.00-2000.00]

643.1595

$\mathrm{C}_{38} \mathrm{H}_{27} \mathrm{O}_{10}=643.1599$

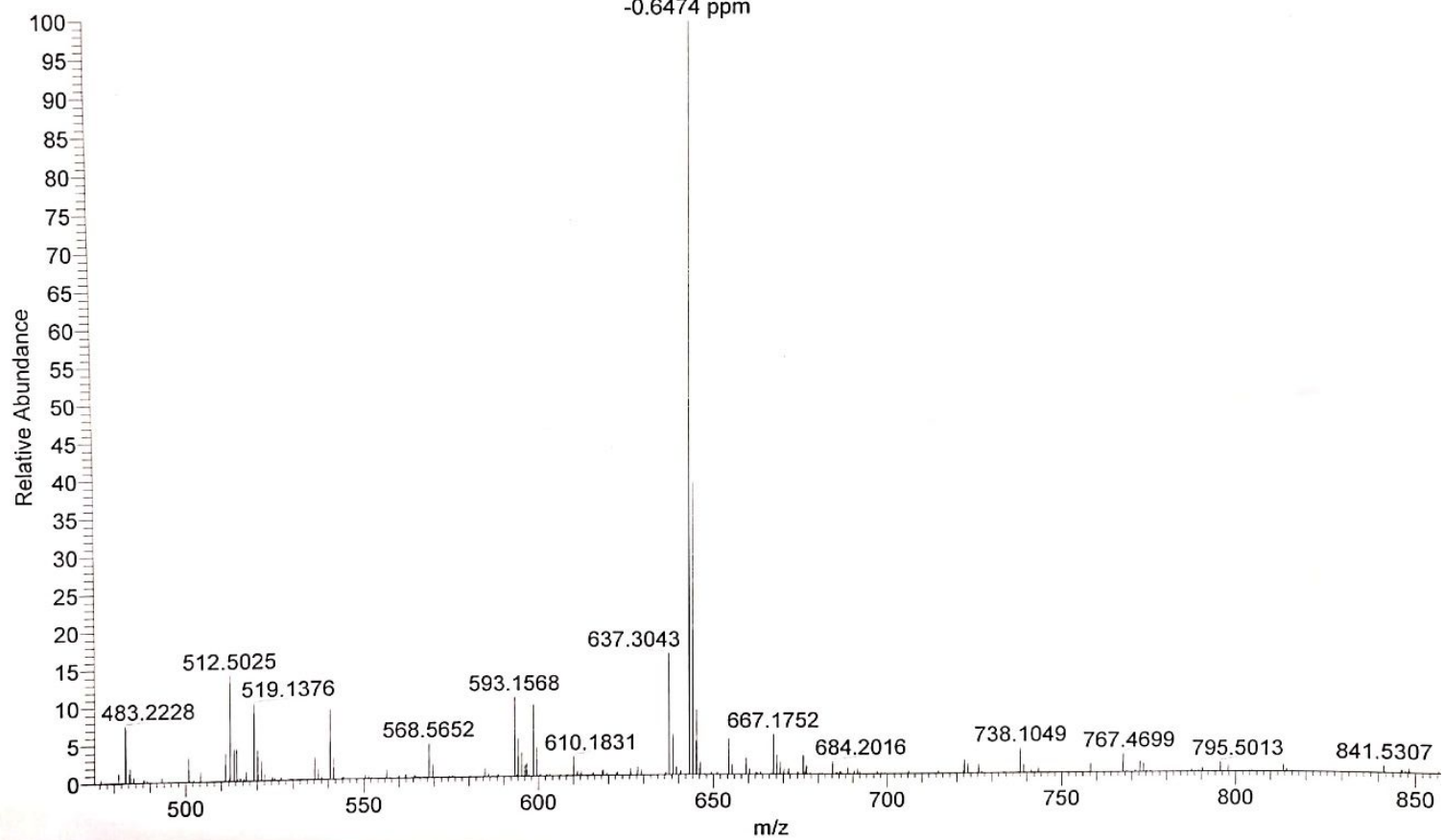

Figure S20. The IR spectrum of compound (-)-2.

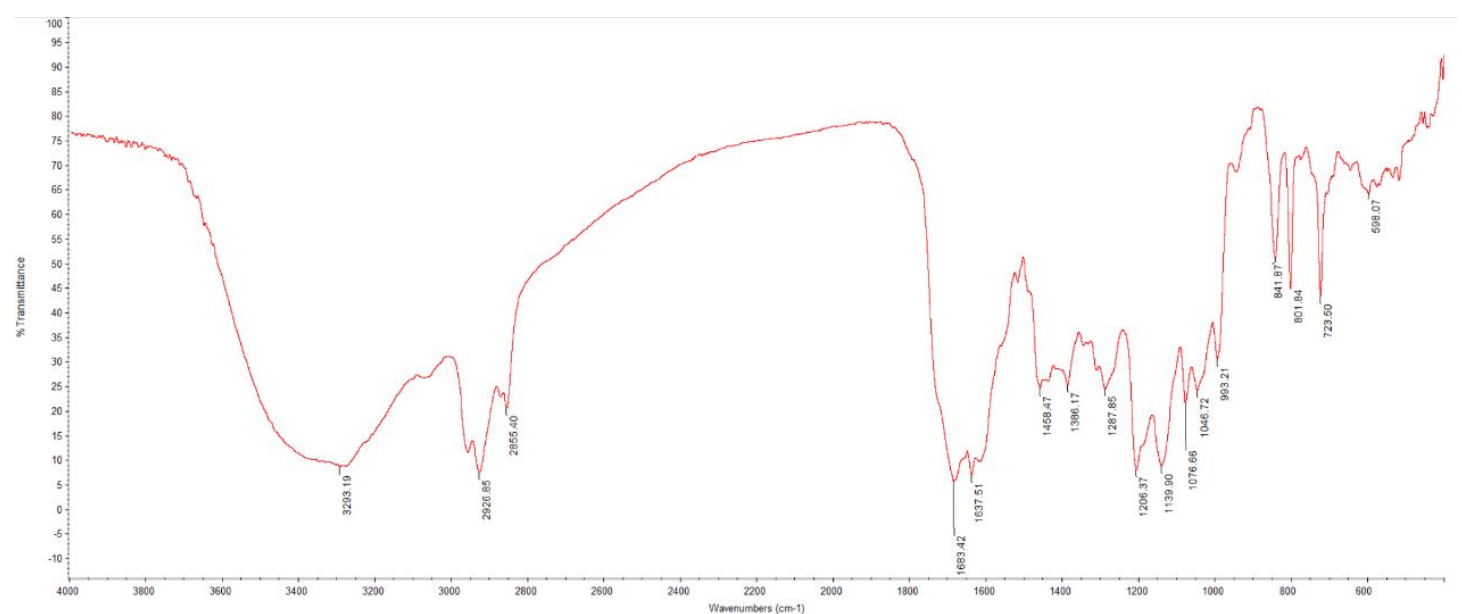


Figure S21. ${ }^{1} \mathrm{H}$ NMR (600 MHz, DMSO) spectrum of compound 3a by synthesis.

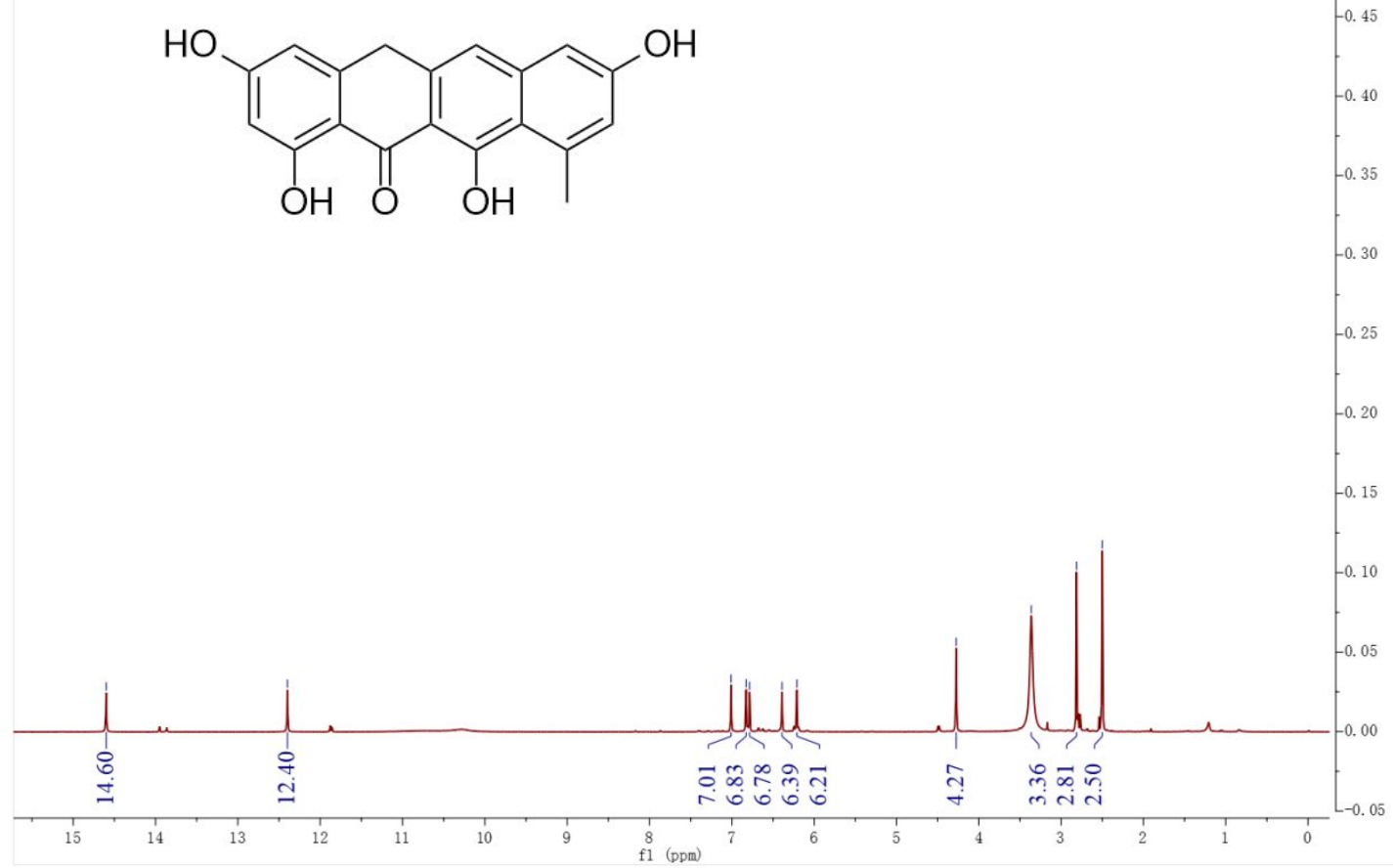

Figure S22. ${ }^{13} \mathrm{C}$ NMR (150 MHz, DMSO) spectrum of compound 3a by synthesis.

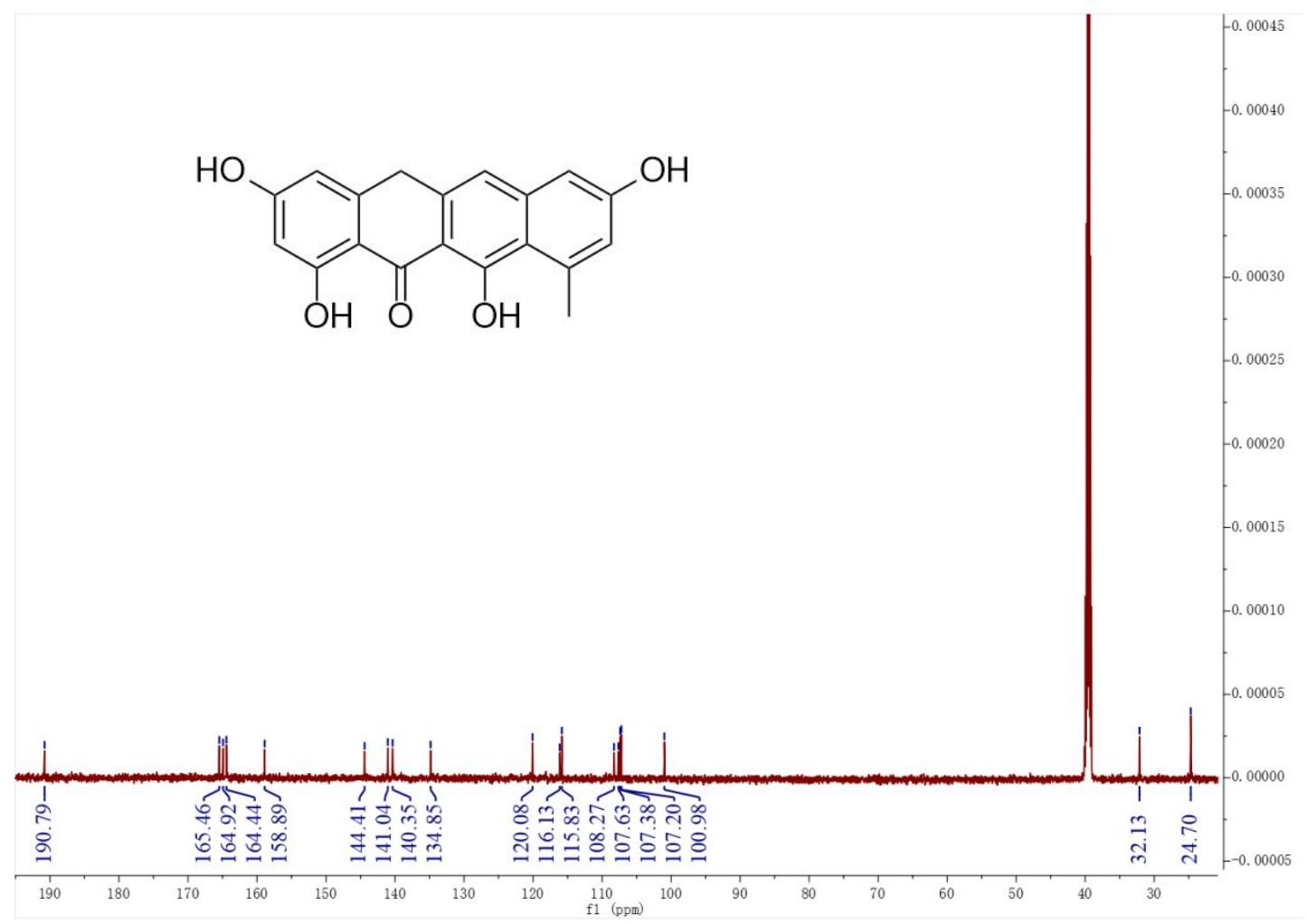


Figure S23. ${ }^{1} \mathrm{H}$ NMR (400 MHz, DMSO) spectrum of $c i s-1$ by synthesis.

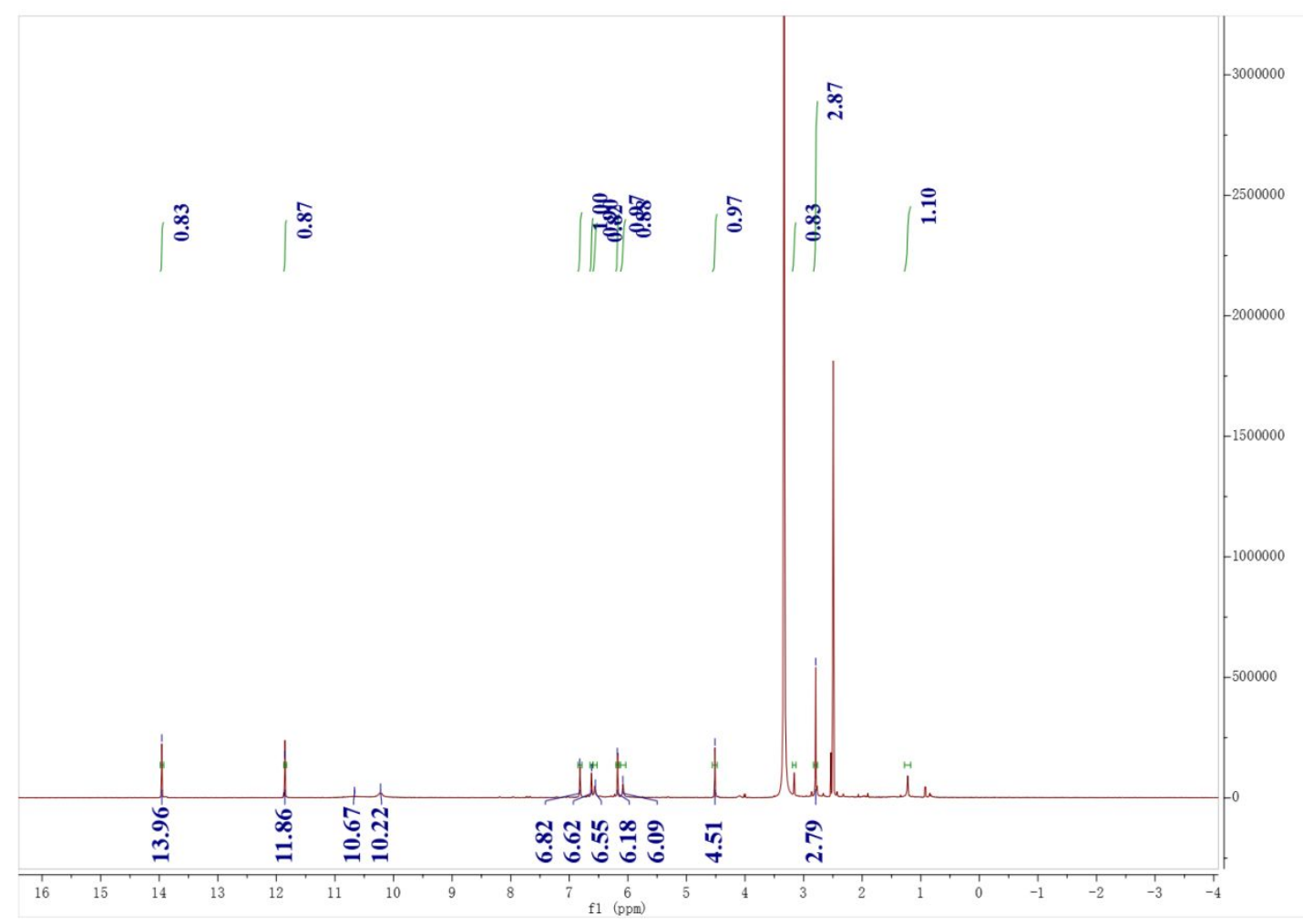

Figure S24. The HRESIMS spectrum of compound cis-1 by synthesis.

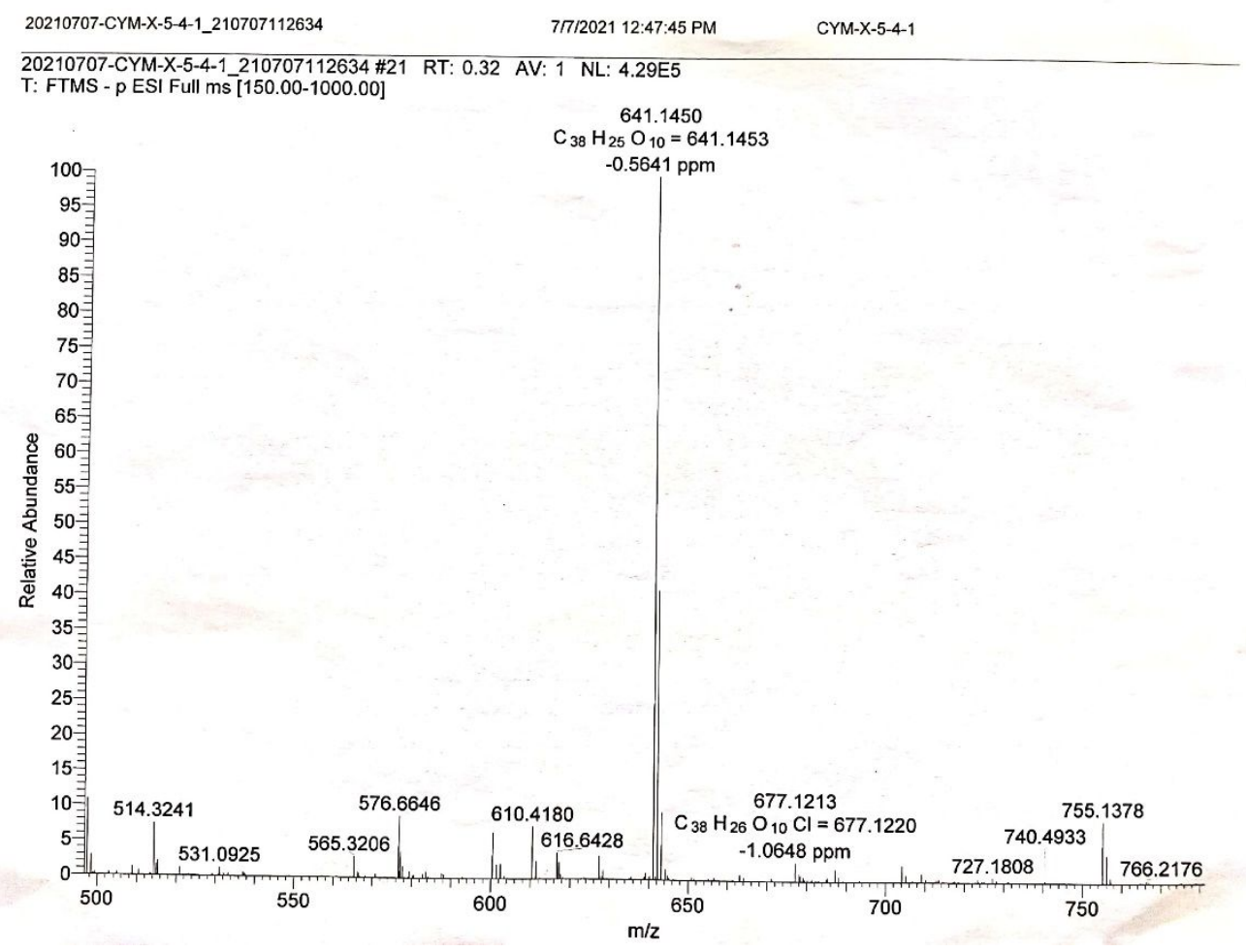


Figure S25. ${ }^{1} \mathrm{H}$ NMR (400 MHz, DMSO) spectrum of (+)-2 by synthesis.

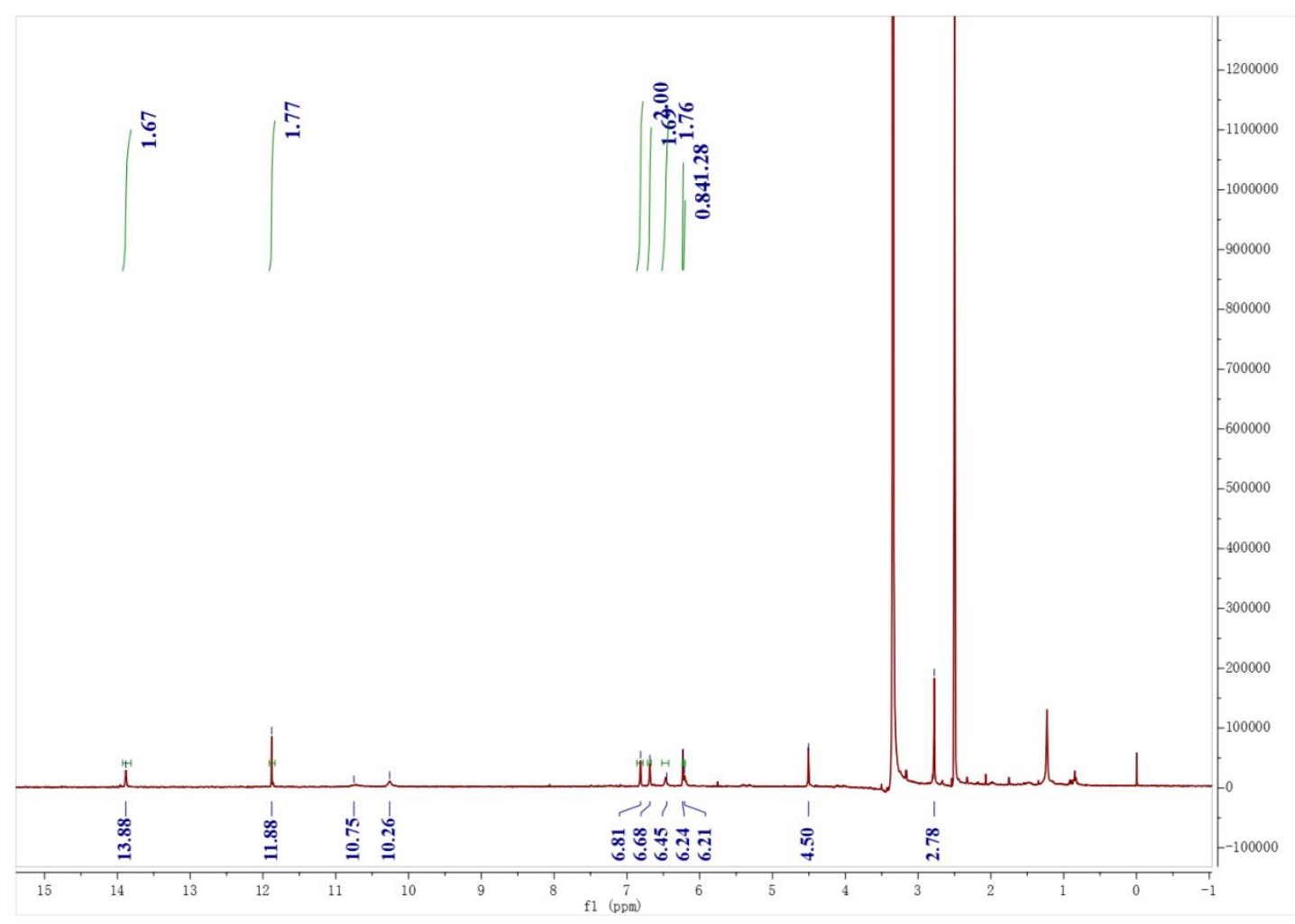

Figure S26. The HRESIMS spectrum of compound ( + )-2 by synthesis.

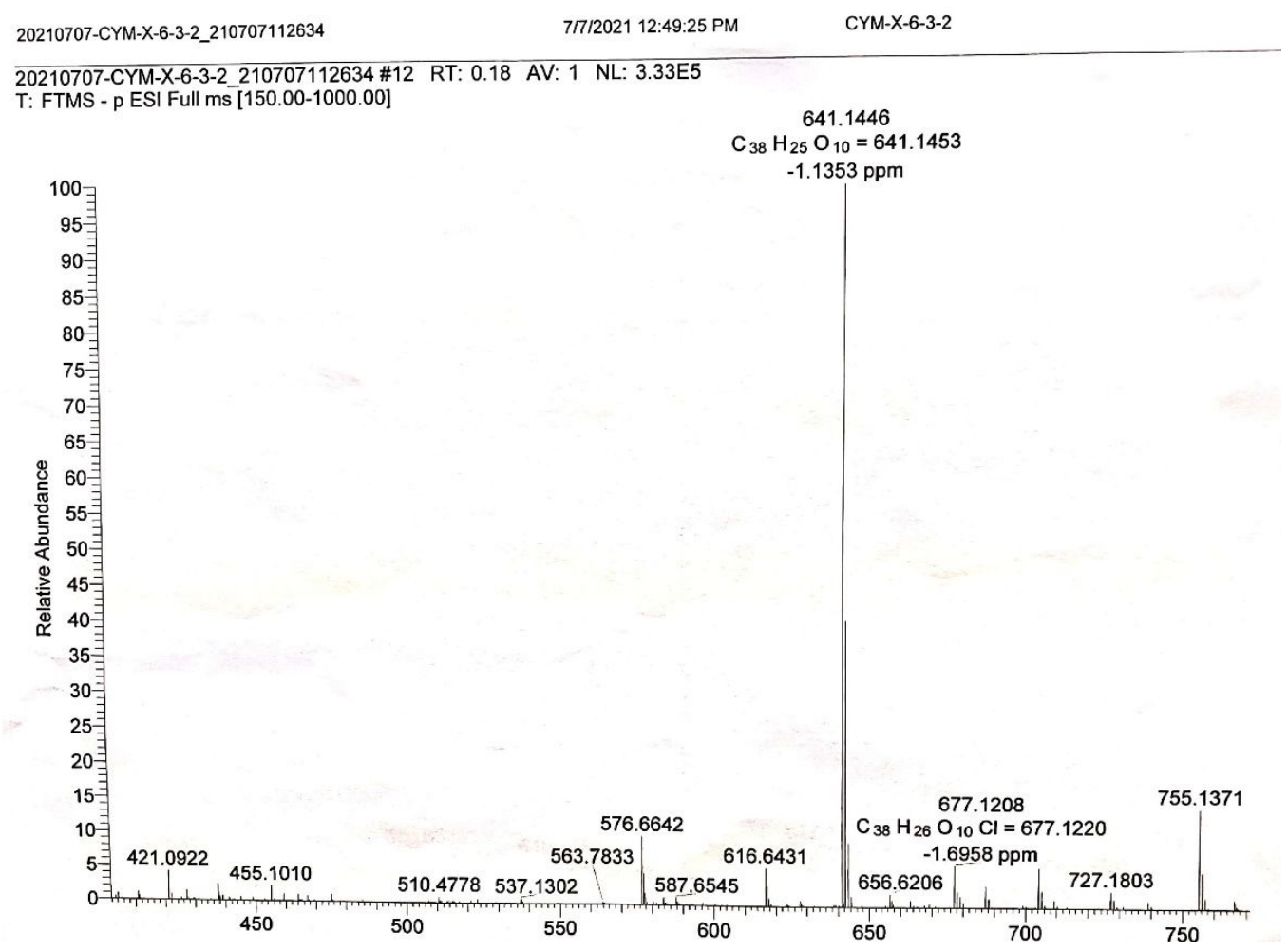


Figure S27. ${ }^{1} \mathrm{H}$ NMR (500 MHz, DMSO) spectrum of compound (-)-2 by synthesis.

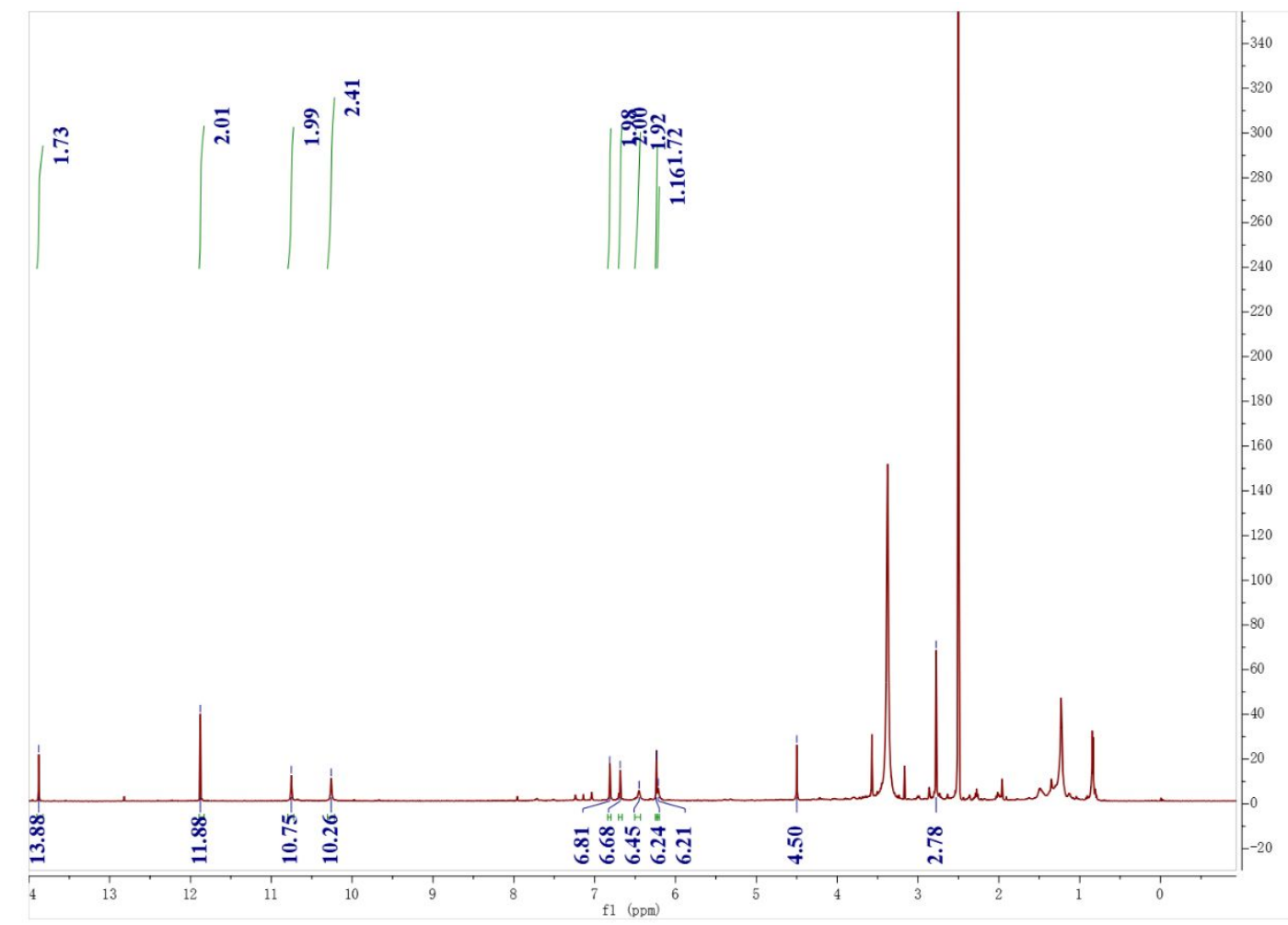

Figure S28. The HRESIMS spectrum of compound (-)-2 by synthesis.

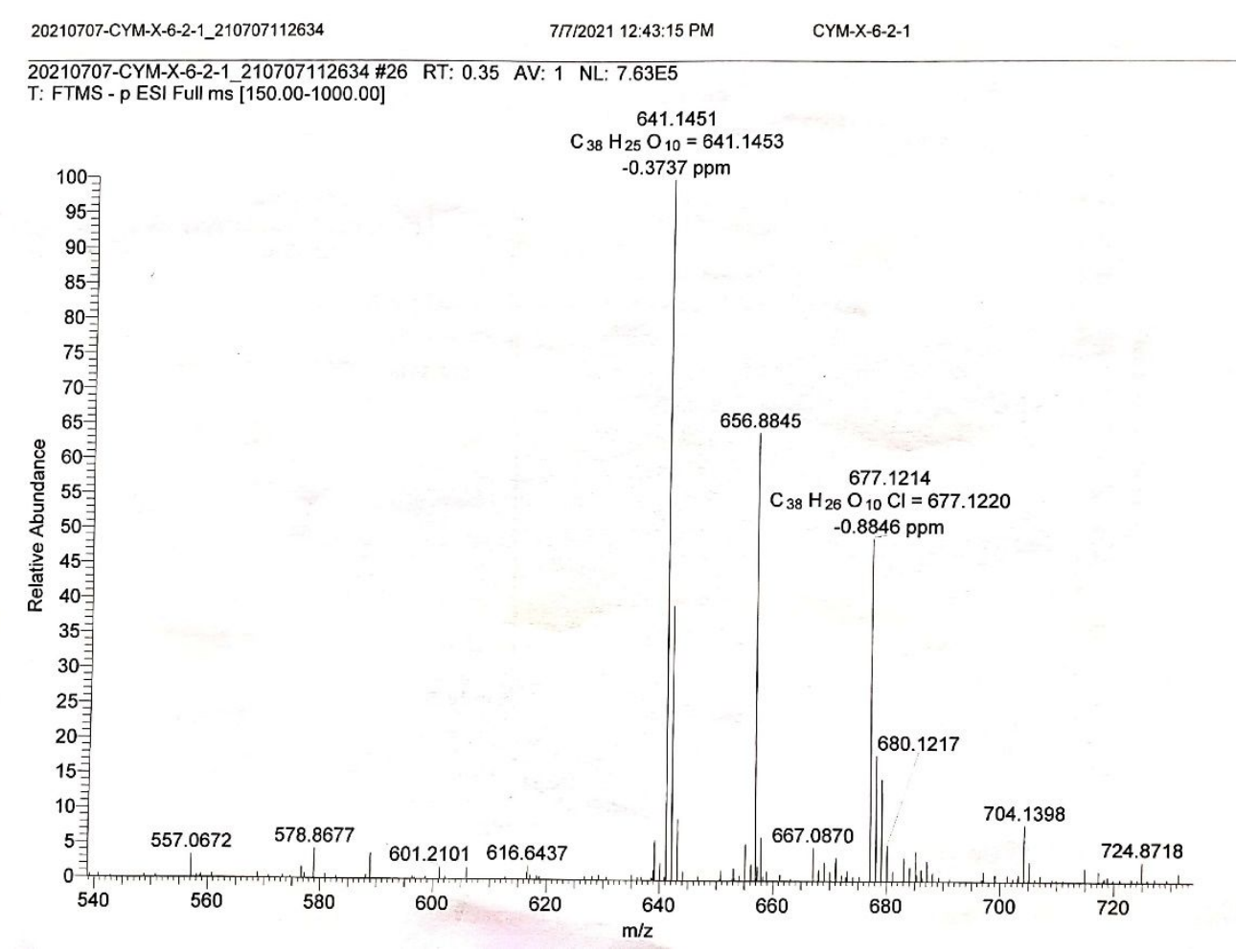


Table S1. Stack spectra and comparison of ${ }^{1} \mathrm{H}$ NMR for compounds 1, (+)-2 and (-)-2
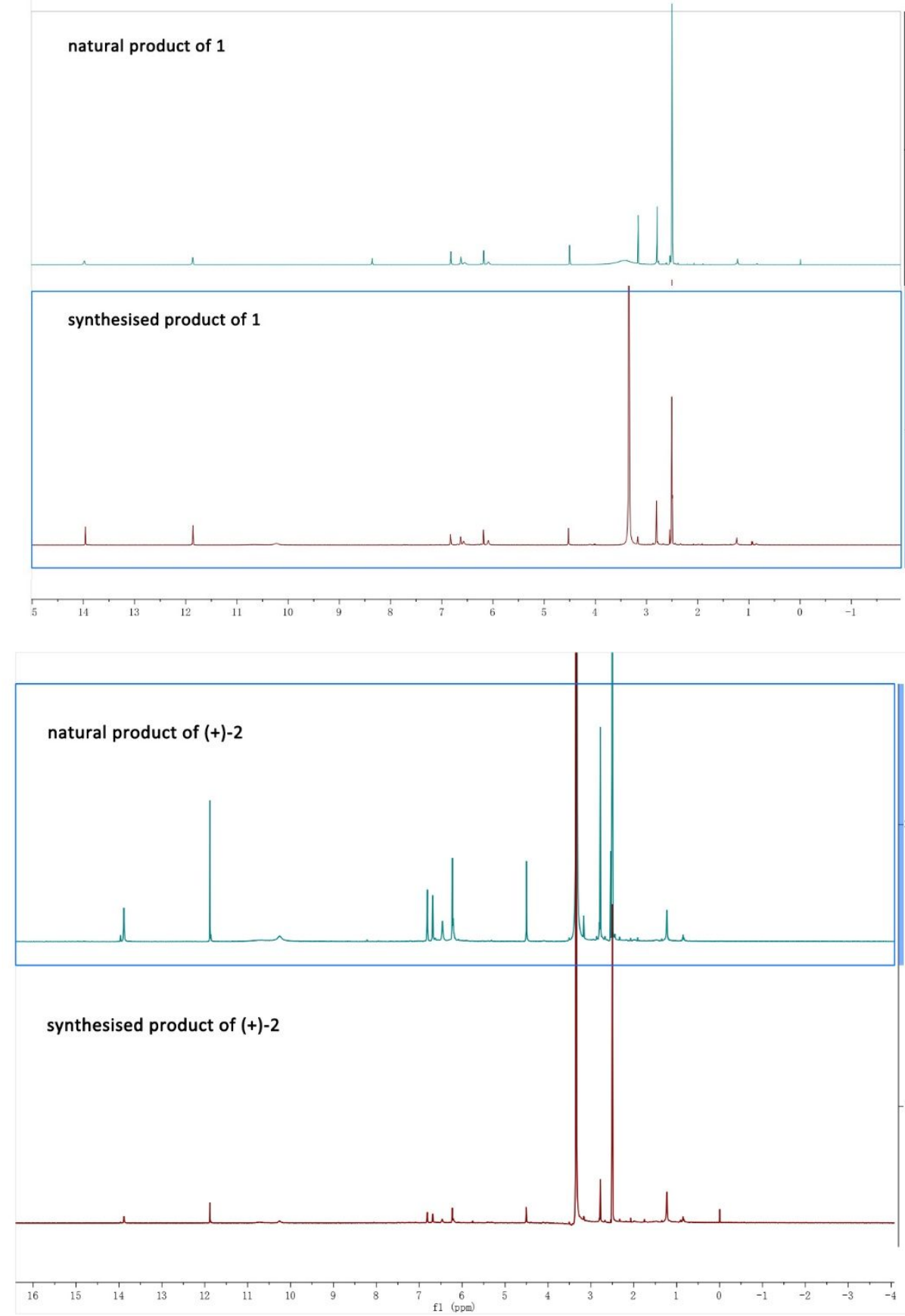
natural product of (-)-2

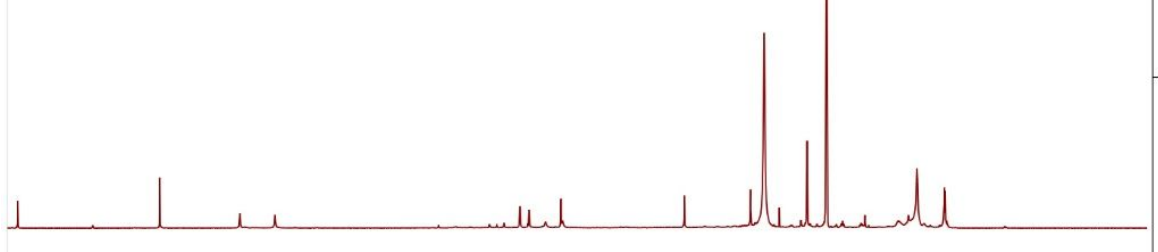

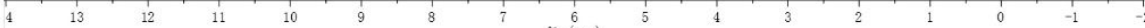

\begin{tabular}{|c|c|c|c|c|c|c|}
\hline \multirow{2}{*}{ Position } & \multicolumn{2}{|r|}{1} & \multicolumn{2}{|r|}{$(+)-2$} & \multicolumn{2}{|r|}{$(-)-2$} \\
\hline & $\mathrm{NP}^{a}$ & semi-synthesized $^{b}$ & $\mathrm{NP}^{b}$ & semi-synthesized ${ }^{b}$ & $\mathrm{NP}^{c}$ & semi-synthesized $^{c}$ \\
\hline $1 / 1^{\prime}$ & $6.09, \mathrm{~s}$ & $6.09, \mathrm{~s}$ & $6.21, \mathrm{~s}$ & $6.21, \mathrm{~s}$ & $6.21, \mathrm{~s}$ & $6.21, \mathrm{~s}$ \\
\hline $3 / 3^{\prime}$ & $6.18, \mathrm{~s}$ & $6.18, \mathrm{~s}$ & $6.24, \mathrm{~s}$ & $6.24, \mathrm{~s}$ & $6.24, \mathrm{~s}$ & $6.24, \mathrm{~s}$ \\
\hline $8 / 8^{\prime}$ & $6.82, \mathrm{~s}$ & $6.82, \mathrm{~s}$ & $6.81, \mathrm{~s}$ & $6.81, \mathrm{~s}$ & $6.81, \mathrm{~s}$ & $6.81, \mathrm{~s}$ \\
\hline $10 / 10^{\prime}$ & $6.62, \mathrm{~s}$ & $6.62, \mathrm{~s}$ & $6.68, \mathrm{~s}$ & $6.68, \mathrm{~s}$ & $6.68, \mathrm{~s}$ & $6.68, \mathrm{~s}$ \\
\hline $11 / 11^{\prime}$ & $6.55, \mathrm{~s}$ & $6.55, \mathrm{~s}$ & $6.45, \mathrm{~s}$ & $6.45, \mathrm{~s}$ & $6.44, \mathrm{~s}$ & $6.45, \mathrm{~s}$ \\
\hline $12 / 12^{\prime}$ & $4.50, \mathrm{~s}$ & $4,51, \mathrm{~s}$ & $4.50, \mathrm{~s}$ & $4.50, \mathrm{~s}$ & $4.50, \mathrm{~s}$ & $4.50, \mathrm{~s}$ \\
\hline $13 / 13^{\prime}$ & $2.79, \mathrm{~s}$ & $2.79, \mathrm{~s}$ & $2.78, \mathrm{~s}$ & $2.78, \mathrm{~s}$ & $2.78, \mathrm{~s}$ & $2.78, \mathrm{~s}$ \\
\hline $2 / 2 '-\mathrm{OH}$ & & 10.67, s & $\begin{array}{l}10.72 \\
\text { s }\end{array}$ & $10.75, \mathrm{~s}$ & & $10.75, \mathrm{~s}$ \\
\hline 4/4'-OH & $\begin{array}{l}\text { 11.86, } \\
\text { s }\end{array}$ & $11.86, \mathrm{~s}$ & $\begin{array}{l}11.88, \\
\text { s }\end{array}$ & $11.88, \mathrm{~s}$ & $\begin{array}{l}11.88, \\
\text { s }\end{array}$ & $11.88, \mathrm{~s}$ \\
\hline 6/6'-OH & $\begin{array}{l}\text { 13.98, } \\
\text { s }\end{array}$ & 19.96, s & $\begin{array}{l}13.88, \\
\text { s }\end{array}$ & $13.88, \mathrm{~s}$ & $\begin{array}{l}13.88, \\
\text { s }\end{array}$ & $13.88, \mathrm{~s}$ \\
\hline 9/9'-OH & & $10.22, \mathrm{~s}$ & $\begin{array}{l}10.24, \\
\text { s }\end{array}$ & $10.26, \mathrm{~s}$ & & $10.26, \mathrm{~s}$ \\
\hline \multicolumn{7}{|c|}{${ }^{a}$ Spectra were recorded at $600 \mathrm{MHz}$ for ${ }^{1} \mathrm{H}$ NMR. } \\
\hline \multicolumn{7}{|c|}{${ }^{b}$ Spectra were recorded at $400 \mathrm{MHz}$ for ${ }^{1} \mathrm{H}$ NMR. } \\
\hline${ }^{c}$ Spectra $v$ & ere recor & led at $500 \mathrm{MHz}$ for ${ }^{1}$ & I NMR. & & & \\
\hline
\end{tabular}


Figure S29. DFT-optimized structures for low-energy conformers of $\left(2 R, 2^{\prime} R\right)-(+)-\mathbf{2}$ at B3LYP/TZVP level in DMSO (CPCM) (Conformer populations were calculated using the Gibbs free energy and Boltzmann population at $298 \mathrm{~K}$ estimated thereof).

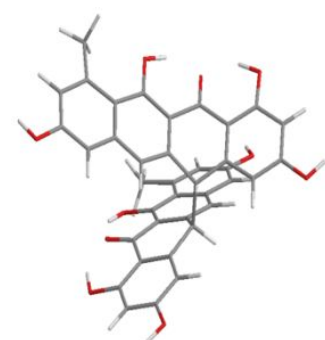

Conf. A $51.9 \%$

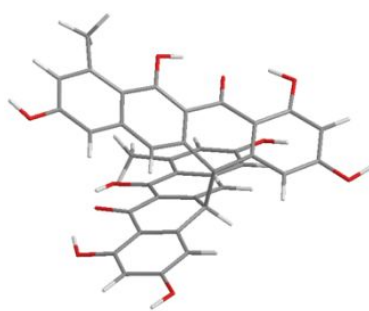

Conf. C $15.6 \%$

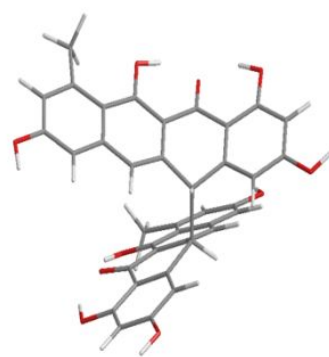

Conf. B $15.6 \%$

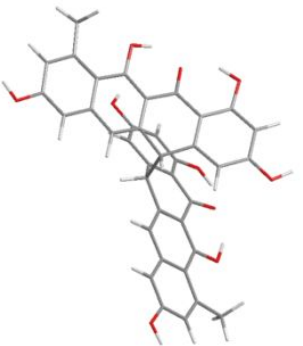

Conf. D $6.7 \%$

Table S2. Harmonic frequencies $\left(\mathrm{cm}^{* *}-2\right)$ of $(+)-2$.

\begin{tabular}{|c|c|c|c|}
\hline \multirow{2}{*}{ Conformations } & \multicolumn{3}{|c|}{ Frequencies } \\
\cline { 2 - 4 } & 1 & 2 & 3 \\
\hline A & 11.6244 & 22.3349 & 26.7318 \\
\hline B & 10.5245 & 20.1622 & 25.1787 \\
\hline C & 10.5246 & 20.1620 & 25.1764 \\
\hline D & 10.4644 & 21.3481 & 26.4872 \\
\hline
\end{tabular}

Table S3. Important thermodynamic parameters (a.u.) and Boltzmann distributions of the optimized (+)-2 at B3LYP/TZVP level of theory with PCM solvent model for DMSO.

\begin{tabular}{|c|c|c|c|}
\hline Conformations & E+ZPE & G & \% \\
\hline A & -2216.262320 & -2216.331379 & 51.9 \\
\hline B & -2216.263060 & -2216.333258 & 15.6 \\
\hline C & -2216.263059 & -2216.333258 & 15.6 \\
\hline D & -2216.262350 & -2216.331923 & 6.7 \\
\hline
\end{tabular}

E+ZPE, G: total energy with zero point energy (ZPE) and Gibbs free energy at B3LYP/TZVP level of theory with PCM solvent model for DMSO. \%: Boltzmann distributions, using the 
relative Gibbs free energies as weighting factors

Table S4. Cartesian coordinates of the low-energy reoptimized conformers of $(2 R$, 2'R)-(+)-2 calculated at B3LYP/TZVP level of theory with PCM solvent model for DMSO

\begin{tabular}{|c|c|c|c|c|}
\hline \multicolumn{2}{|c|}{ Conformer A } & \multicolumn{3}{|c|}{$\begin{array}{c}\text { Standard Orientation } \\
\text { (Ångstroms) }\end{array}$} \\
\hline I & Atom & $\mathrm{X}$ & $\mathrm{Y}$ & $\mathrm{Z}$ \\
\hline 1 & $\mathrm{C}$ & 3.05599 & 1.99247 & 3.88753 \\
\hline 2 & $\mathrm{C}$ & 3.79523 & 2.571 & 2.86421 \\
\hline 3 & $\mathrm{C}$ & 3.25855 & 2.61054 & 1.58039 \\
\hline 4 & $\mathrm{C}$ & 1.98581 & 2.08068 & 1.31952 \\
\hline 5 & $\mathrm{C}$ & 1.25215 & 1.47337 & 2.34987 \\
\hline 6 & $\mathrm{C}$ & 1.79952 & 1.4452 & 3.64392 \\
\hline 7 & $\mathrm{C}$ & 1.39946 & 2.16497 & -0.03511 \\
\hline 8 & $\mathrm{C}$ & -0.04689 & 1.90014 & -0.22264 \\
\hline 9 & $\mathrm{C}$ & -0.76985 & 1.33627 & 0.83135 \\
\hline 10 & $\mathrm{C}$ & -0.07838 & 0.79001 & 2.07428 \\
\hline 11 & $\mathrm{C}$ & -0.69694 & 2.26945 & -1.4196 \\
\hline 12 & $\mathrm{C}$ & -2.10648 & 2.12458 & -1.58986 \\
\hline 13 & $\mathrm{C}$ & -2.82635 & 1.60897 & -0.47929 \\
\hline 14 & $\mathrm{C}$ & -2.16137 & 1.23989 & 0.70044 \\
\hline 15 & $\mathrm{C}$ & -2.86014 & 2.45731 & -2.76673 \\
\hline 16 & $\mathrm{C}$ & -4.25651 & 2.27901 & -2.79579 \\
\hline 17 & $\mathrm{C}$ & -4.91756 & 1.778 & -1.68768 \\
\hline 18 & $\mathrm{C}$ & -4.21663 & 1.44475 & -0.54121 \\
\hline 19 & $\mathrm{O}$ & 2.09988 & 2.51442 & -0.98377 \\
\hline 20 & $\mathrm{O}$ & -6.26814 & 1.59419 & -1.68445 \\
\hline 21 & $\mathrm{C}$ & -2.21334 & 3.0054 & -4.01508 \\
\hline 22 & $\mathrm{O}$ & 0.03387 & 2.79815 & -2.46043 \\
\hline 23 & $\mathrm{O}$ & 4.04045 & 3.18653 & 0.61553 \\
\hline 24 & $\mathrm{O}$ & 3.53496 & 1.92578 & 5.1615 \\
\hline 25 & $\mathrm{C}$ & -3.79606 & -2.57513 & 2.85939 \\
\hline 26 & $\mathrm{C}$ & -3.05711 & -1.99808 & 3.88376 \\
\hline 27 & $\mathrm{C}$ & -1.80057 & -1.45046 & 3.64131 \\
\hline 28 & $\mathrm{C}$ & -1.25283 & -1.47676 & 2.34738 \\
\hline 29 & $\mathrm{C}$ & -1.98619 & -2.08258 & 1.31593 \\
\hline 30 & $\mathrm{C}$ & -3.25901 & -2.61282 & 1.57567 \\
\hline 31 & $\mathrm{C}$ & 0.07778 & -0.793 & 2.07316 \\
\hline 32 & $\mathrm{C}$ & 0.76961 & -1.33747 & 0.82965 \\
\hline 33 & $\mathrm{C}$ & 0.04696 & -1.89982 & -0.22537 \\
\hline
\end{tabular}




\begin{tabular}{|c|c|c|c|c|}
\hline 34 & $\mathrm{C}$ & -1.39945 & -2.16492 & -0.03864 \\
\hline 35 & $\mathrm{C}$ & 2.16117 & -1.2409 & 0.69928 \\
\hline 36 & $\mathrm{C}$ & 2.82649 & -1.60827 & -0.48079 \\
\hline 37 & $\mathrm{C}$ & 2.10694 & -2.12228 & -1.59232 \\
\hline 38 & $\mathrm{C}$ & 0.69735 & -2.2674 & -1.42267 \\
\hline 39 & $\mathrm{C}$ & 4.21679 & -1.44397 & -0.54208 \\
\hline 40 & $\mathrm{C}$ & 4.91805 & -1.77557 & -1.68882 \\
\hline 41 & $\mathrm{C}$ & 4.25732 & -2.27497 & -2.79785 \\
\hline 42 & $\mathrm{C}$ & 2.86095 & -2.45331 & -2.76945 \\
\hline 43 & $\mathrm{O}$ & -2.09959 & -2.51299 & -0.98801 \\
\hline 44 & $\mathrm{C}$ & 2.21451 & -2.9996 & -4.01878 \\
\hline 45 & $\mathrm{O}$ & -0.03316 & -2.7946 & -2.46448 \\
\hline 46 & $\mathrm{O}$ & -4.04063 & -3.18742 & 0.60975 \\
\hline 47 & $\mathrm{O}$ & 6.26863 & -1.59176 & -1.68493 \\
\hline 48 & $\mathrm{O}$ & -3.53646 & -1.93323 & 5.15768 \\
\hline 49 & $\mathrm{H}$ & 0.71929 & -1.08291 & 2.91948 \\
\hline 50 & $\mathrm{H}$ & -0.72014 & 1.0787 & 2.92083 \\
\hline 51 & $\mathrm{H}$ & 4.78006 & 2.99174 & 3.04098 \\
\hline 52 & $\mathrm{H}$ & 1.25792 & 0.9892 & 4.47051 \\
\hline 53 & $\mathrm{H}$ & -2.74978 & 0.85248 & 1.53308 \\
\hline 54 & $\mathrm{H}$ & -4.8134 & 2.53643 & -3.69237 \\
\hline 55 & $\mathrm{H}$ & -4.77168 & 1.04677 & 0.3056 \\
\hline 56 & $\mathrm{H}$ & -6.61702 & 1.84367 & -2.55602 \\
\hline 57 & $\mathrm{H}$ & -1.74284 & 3.97209 & -3.81081 \\
\hline 58 & $\mathrm{H}$ & -1.48024 & 2.2959 & -4.41122 \\
\hline 59 & $\mathrm{H}$ & -2.94943 & 3.1731 & -4.80942 \\
\hline 60 & $\mathrm{H}$ & 0.98749 & 2.7741 & -2.22698 \\
\hline 61 & $\mathrm{H}$ & 3.58608 & 3.07628 & -0.24995 \\
\hline 62 & $\mathrm{H}$ & 4.40403 & 2.36195 & 5.19226 \\
\hline 63 & $\mathrm{H}$ & -4.78094 & -2.99612 & 3.03527 \\
\hline 64 & $\mathrm{H}$ & -1.25921 & -0.99565 & 4.46871 \\
\hline 65 & $\mathrm{H}$ & 2.74933 & -0.85469 & 1.53265 \\
\hline 66 & $\mathrm{H}$ & 4.77159 & -1.04721 & 0.30547 \\
\hline 67 & $\mathrm{H}$ & 4.81447 & -2.53109 & -3.69463 \\
\hline 68 & $\mathrm{H}$ & 2.95082 & -3.16615 & -4.81315 \\
\hline 69 & $\mathrm{H}$ & 1.74394 & -3.96659 & -3.81604 \\
\hline 70 & $\mathrm{H}$ & 1.48152 & -2.28952 & -4.4141 \\
\hline 71 & $\mathrm{H}$ & -0.98684 & -2.77088 & -2.23127 \\
\hline 72 & $\mathrm{H}$ & -3.58601 & -3.07592 & -0.25543 \\
\hline 73 & $\mathrm{H}$ & 6.61777 & -1.83998 & -2.55676 \\
\hline 74 & $\mathrm{H}$ & -4.40553 & -2.36944 & 5.18756 \\
\hline
\end{tabular}




\begin{tabular}{|c|c|c|c|c|}
\hline \multicolumn{2}{|c|}{ Conformer B } & \multicolumn{3}{|c|}{$\begin{array}{c}\text { Standard Orientation } \\
\text { (Ångstroms) }\end{array}$} \\
\hline I & Atom & $\mathrm{X}$ & $\mathrm{Y}$ & Z \\
\hline 1 & $\mathrm{C}$ & 1.68038 & -0.12581 & 5.05522 \\
\hline 2 & $\mathrm{C}$ & 1.46488 & 1.2459 & 5.05817 \\
\hline 3 & $\mathrm{C}$ & 0.72234 & 1.81651 & 4.0282 \\
\hline 4 & $\mathrm{C}$ & 0.19111 & 1.02064 & 3.00189 \\
\hline 5 & $\mathrm{C}$ & 0.43255 & -0.3613 & 2.98729 \\
\hline 6 & $\mathrm{C}$ & 1.1767 & -0.92874 & 4.03587 \\
\hline 7 & $\mathrm{C}$ & -0.63535 & 1.62343 & 1.93462 \\
\hline 8 & $\mathrm{C}$ & -1.44187 & 0.74748 & 1.05164 \\
\hline 9 & $\mathrm{C}$ & -1.21713 & -0.63088 & 1.08293 \\
\hline 10 & $\mathrm{C}$ & -0.03362 & -1.23244 & 1.83126 \\
\hline 11 & $\mathrm{C}$ & -2.4609 & 1.28748 & 0.23852 \\
\hline 12 & $\mathrm{C}$ & -3.31782 & 0.46084 & -0.54858 \\
\hline 13 & $\mathrm{C}$ & -3.10661 & -0.94128 & -0.45435 \\
\hline 14 & $\mathrm{C}$ & -2.08703 & -1.45877 & 0.36047 \\
\hline 15 & $\mathrm{C}$ & -4.37034 & 0.91126 & -1.41422 \\
\hline 16 & $\mathrm{C}$ & -5.14893 & -0.01637 & -2.12967 \\
\hline 17 & $\mathrm{C}$ & -4.90921 & -1.37301 & -2.01208 \\
\hline 18 & $\mathrm{C}$ & -3.90094 & -1.83963 & -1.18371 \\
\hline 19 & $\mathrm{O}$ & -0.69539 & 2.84705 & 1.82654 \\
\hline 20 & $\mathrm{O}$ & -5.69119 & -2.22333 & -2.73548 \\
\hline 21 & $\mathrm{C}$ & -4.69745 & 2.37094 & -1.60807 \\
\hline 22 & $\mathrm{O}$ & -2.65426 & 2.65039 & 0.19852 \\
\hline 23 & $\mathrm{O}$ & 0.55269 & 3.1737 & 4.08561 \\
\hline 24 & $\mathrm{O}$ & 2.40119 & -0.73521 & 6.03799 \\
\hline 25 & $\mathrm{C}$ & 0.11975 & -5.24038 & -1.27058 \\
\hline 26 & $\mathrm{C}$ & 0.49484 & -5.29414 & 0.06533 \\
\hline 27 & $\mathrm{C}$ & 0.86458 & -4.14206 & 0.75354 \\
\hline 28 & $\mathrm{C}$ & 0.87313 & -2.89753 & 0.10085 \\
\hline 29 & $\mathrm{C}$ & 0.52473 & -2.84503 & -1.25709 \\
\hline 30 & $\mathrm{C}$ & 0.13122 & -4.0132 & -1.92773 \\
\hline 31 & $\mathrm{C}$ & 1.18206 & -1.63158 & 0.88564 \\
\hline 32 & $\mathrm{C}$ & 1.66044 & -0.49259 & -0.00575 \\
\hline 33 & $\mathrm{C}$ & 1.28583 & -0.40436 & -1.34882 \\
\hline 34 & $\mathrm{C}$ & 0.58239 & -1.55148 & -1.97051 \\
\hline 35 & $\mathrm{C}$ & 2.45937 & 0.52821 & 0.52554 \\
\hline 36 & $\mathrm{C}$ & 2.81521 & 1.66148 & -0.22227 \\
\hline 37 & $\mathrm{C}$ & 2.39357 & 1.80563 & -1.57098 \\
\hline 38 & $\mathrm{C}$ & 1.62739 & 0.73098 & -2.11402 \\
\hline 39 & $\mathrm{C}$ & 3.58593 & 2.65466 & 0.39679 \\
\hline
\end{tabular}




\begin{tabular}{|l|l|l|l|l|}
\hline 40 & $\mathrm{C}$ & 3.94922 & 3.79948 & -0.29179 \\
\hline 41 & $\mathrm{C}$ & 3.55605 & 3.98464 & -1.60594 \\
\hline 42 & $\mathrm{C}$ & 2.78204 & 3.00587 & -2.25815 \\
\hline 43 & $\mathrm{O}$ & 0.1002 & -1.45618 & -3.09785 \\
\hline 44 & $\mathrm{C}$ & 2.39073 & 3.28334 & -3.68894 \\
\hline 45 & $\mathrm{O}$ & 1.22818 & 0.81549 & -3.4293 \\
\hline 46 & $\mathrm{O}$ & -0.26462 & -4.02537 & -3.23833 \\
\hline 47 & $\mathrm{O}$ & 4.6987 & 4.72798 & 0.367 \\
\hline 48 & $\mathrm{O}$ & 0.50076 & -6.4685 & 0.75619 \\
\hline 49 & $\mathrm{H}$ & 2.045 & -1.89802 & 1.51498 \\
\hline 50 & $\mathrm{H}$ & -0.42045 & -2.1451 & 2.31012 \\
\hline 51 & $\mathrm{H}$ & 1.86115 & 1.88286 & 5.84268 \\
\hline 52 & $\mathrm{H}$ & 1.3789 & -1.99776 & 4.06382 \\
\hline 53 & $\mathrm{H}$ & -1.95979 & -2.54022 & 0.4177 \\
\hline 54 & $\mathrm{H}$ & -5.94663 & 0.32068 & -2.78766 \\
\hline 55 & $\mathrm{H}$ & -3.72365 & -2.90902 & -1.10608 \\
\hline 56 & $\mathrm{H}$ & -5.37677 & -3.13187 & -2.60006 \\
\hline 57 & $\mathrm{H}$ & -5.01881 & 2.82107 & -0.66376 \\
\hline 58 & $\mathrm{H}$ & -3.83508 & 2.90701 & -2.01636 \\
\hline 59 & $\mathrm{H}$ & -5.51936 & 2.50807 & -2.31987 \\
\hline 60 & $\mathrm{H}$ & -1.95348 & 3.09053 & 0.72703 \\
\hline 61 & $\mathrm{H}$ & 0.08594 & 3.45719 & 3.26736 \\
\hline 62 & $\mathrm{H}$ & 2.67061 & -0.06497 & 6.68973 \\
\hline 63 & $\mathrm{H}$ & -0.1813 & -6.13099 & -1.81311 \\
\hline 64 & $\mathrm{H}$ & 1.13689 & -4.22427 & 1.80418 \\
\hline 65 & $\mathrm{H}$ & 2.80644 & 0.45213 & 1.55672 \\
\hline 66 & $\mathrm{H}$ & 3.90816 & 2.55038 & 1.43065 \\
\hline 67 & $\mathrm{H}$ & 3.84337 & 4.88672 & -2.13884 \\
\hline 68 & $\mathrm{H}$ & 2.76056 & 4.25845 & -4.02555 \\
\hline 69 & $\mathrm{H}$ & 2.82364 & 2.533 & -4.35763 \\
\hline 70 & $\mathrm{H}$ & 1.3014 & 3.3084 & -3.79139 \\
\hline 71 & $\mathrm{H}$ & 0.65021 & 0.0502 & -3.63983 \\
\hline 73 & $\mathrm{H}$ & -0.25973 & -3.09763 & -3.56673 \\
\hline 74 & $\mathrm{H}$ & 4.84953 & 5.48223 & -0.22632 \\
\hline & & 0.27773 & -7.19323 & 0.14677 \\
\hline
\end{tabular}

\begin{tabular}{|c|l|c|c|c|}
\hline \multicolumn{2}{|c|}{ Conformer C } & \multicolumn{3}{|c|}{$\begin{array}{c}\text { Standard Orientation } \\
\text { (̊ngstroms) }\end{array}$} \\
\hline $\mathrm{I}$ & Atom & $\mathrm{X}$ & $\mathrm{Y}$ & $\mathrm{Z}$ \\
\hline 1 & $\mathrm{C}$ & 1.69532 & -0.14238 & 5.03812 \\
\hline 2 & $\mathrm{C}$ & 1.49735 & 1.23204 & 5.03292 \\
\hline 3 & $\mathrm{C}$ & 0.76148 & 1.80575 & 3.99981 \\
\hline
\end{tabular}




\begin{tabular}{|c|c|c|c|c|}
\hline 4 & $\mathrm{C}$ & 0.2168 & 1.00989 & 2.98042 \\
\hline 5 & $\mathrm{C}$ & 0.44486 & -0.37433 & 2.97155 \\
\hline 6 & $\mathrm{C}$ & 1.18347 & -0.94472 & 4.0223 \\
\hline 7 & $\mathrm{C}$ & -0.60487 & 1.61578 & 1.91117 \\
\hline 8 & $\mathrm{C}$ & -1.42018 & 0.74364 & 1.03261 \\
\hline 9 & $\mathrm{C}$ & -1.20557 & -0.63643 & 1.06832 \\
\hline 10 & $\mathrm{C}$ & -0.02509 & -1.24399 & 1.81535 \\
\hline 11 & $\mathrm{C}$ & -2.43752 & 1.28899 & 0.22095 \\
\hline 12 & $\mathrm{C}$ & -3.30606 & 0.46606 & -0.557 \\
\hline 13 & $\mathrm{C}$ & -3.10691 & -0.9365 & -0.45328 \\
\hline 14 & $\mathrm{C}$ & -2.08477 & -1.46029 & 0.35356 \\
\hline 15 & $\mathrm{C}$ & -4.35973 & 0.91987 & -1.42161 \\
\hline 16 & $\mathrm{C}$ & -5.1568 & -0.00448 & -2.12354 \\
\hline 17 & $\mathrm{C}$ & -4.93024 & -1.36321 & -1.98796 \\
\hline 18 & $\mathrm{C}$ & -3.91911 & -1.82938 & -1.16513 \\
\hline 19 & $\mathrm{O}$ & -0.65217 & 2.83963 & 1.79955 \\
\hline 20 & $\mathrm{O}$ & -5.68442 & -2.28292 & -2.65369 \\
\hline 21 & $\mathrm{C}$ & -4.66725 & 2.38316 & -1.62533 \\
\hline 22 & $\mathrm{O}$ & -2.61972 & 2.65327 & 0.17575 \\
\hline 23 & $\mathrm{O}$ & 0.61631 & 3.16644 & 4.04404 \\
\hline 24 & $\mathrm{O}$ & 2.40815 & -0.75503 & 6.02452 \\
\hline 25 & $\mathrm{C}$ & 0.14037 & -5.25218 & -1.29542 \\
\hline 26 & $\mathrm{C}$ & 0.49881 & -5.30508 & 0.04501 \\
\hline 27 & $\mathrm{C}$ & 0.86364 & -4.15307 & 0.73586 \\
\hline 28 & $\mathrm{C}$ & 0.87969 & -2.90875 & 0.08266 \\
\hline 29 & $\mathrm{C}$ & 0.54333 & -2.85659 & -1.27837 \\
\hline 30 & $\mathrm{C}$ & 0.16068 & -4.02567 & -1.95348 \\
\hline 31 & $\mathrm{C}$ & 1.18944 & -1.64438 & 0.86879 \\
\hline 32 & $\mathrm{C}$ & 1.67257 & -0.50426 & -0.01963 \\
\hline 33 & $\mathrm{C}$ & 1.30666 & -0.41639 & -1.3649 \\
\hline 34 & $\mathrm{C}$ & 0.60784 & -1.5635 & -1.99197 \\
\hline 35 & $\mathrm{C}$ & 2.46496 & 0.51914 & 0.5178 \\
\hline 36 & $\mathrm{C}$ & 2.81874 & 1.65778 & -0.22355 \\
\hline 37 & $\mathrm{C}$ & 2.40887 & 1.7977 & -1.57701 \\
\hline 38 & $\mathrm{C}$ & 1.65172 & 0.71999 & -2.12697 \\
\hline 39 & $\mathrm{C}$ & 3.57276 & 2.65934 & 0.40756 \\
\hline 40 & $\mathrm{C}$ & 3.93208 & 3.80707 & -0.28102 \\
\hline 41 & $\mathrm{C}$ & 3.55553 & 3.98063 & -1.60005 \\
\hline 42 & $\mathrm{C}$ & 2.79808 & 2.99741 & -2.26199 \\
\hline 43 & $\mathrm{O}$ & 0.136 & -1.46645 & -3.12355 \\
\hline 44 & $\mathrm{C}$ & 2.42635 & 3.27323 & -3.6976 \\
\hline 45 & $\mathrm{O}$ & 1.26227 & 0.80387 & -3.44522 \\
\hline
\end{tabular}




\begin{tabular}{|c|c|c|c|c|}
\hline 46 & $\mathrm{O}$ & -0.21057 & -4.04054 & -3.27106 \\
\hline 47 & $\mathrm{O}$ & 4.65896 & 4.79728 & 0.30972 \\
\hline 48 & $\mathrm{O}$ & 0.49469 & -6.47884 & 0.73714 \\
\hline 49 & $\mathrm{H}$ & 2.05151 & -1.9134 & 1.49836 \\
\hline 50 & $\mathrm{H}$ & -0.41439 & -2.15589 & 2.29348 \\
\hline 51 & $\mathrm{H}$ & 1.90196 & 1.86793 & 5.81401 \\
\hline 52 & $\mathrm{H}$ & 1.37401 & -2.01589 & 4.05466 \\
\hline 53 & $\mathrm{H}$ & -1.96675 & -2.54306 & 0.41066 \\
\hline 54 & $\mathrm{H}$ & -5.95087 & 0.35353 & -2.77283 \\
\hline 55 & $\mathrm{H}$ & -3.76808 & -2.90426 & -1.09171 \\
\hline 56 & $\mathrm{H}$ & -6.32756 & -1.81262 & -3.20944 \\
\hline 57 & $\mathrm{H}$ & -4.98227 & 2.8438 & -0.68397 \\
\hline 58 & $\mathrm{H}$ & -3.79795 & 2.90467 & -2.03776 \\
\hline 59 & $\mathrm{H}$ & -5.48721 & 2.52674 & -2.33805 \\
\hline 60 & $\mathrm{H}$ & -1.90902 & 3.09003 & 0.69362 \\
\hline 61 & $\mathrm{H}$ & 0.12526 & 3.44681 & 3.23853 \\
\hline 62 & $\mathrm{H}$ & 2.65701 & -0.09179 & 6.69127 \\
\hline 63 & $\mathrm{H}$ & -0.15516 & -6.14257 & -1.84137 \\
\hline 64 & $\mathrm{H}$ & 1.1253 & -4.23557 & 1.78904 \\
\hline 65 & $\mathrm{H}$ & 2.80542 & 0.43943 & 1.55063 \\
\hline 66 & $\mathrm{H}$ & 3.87386 & 2.54097 & 1.44504 \\
\hline 67 & $\mathrm{H}$ & 3.85151 & 4.89174 & -2.11496 \\
\hline 68 & $\mathrm{H}$ & 2.80171 & 4.24756 & -4.03043 \\
\hline 69 & $\mathrm{H}$ & 2.86748 & 2.52172 & -4.3596 \\
\hline 70 & $\mathrm{H}$ & 1.33851 & 3.29857 & -3.81474 \\
\hline 71 & $\mathrm{H}$ & 0.69366 & 0.03341 & -3.66249 \\
\hline 72 & $\mathrm{H}$ & -0.23113 & -3.11092 & -3.59222 \\
\hline 73 & $\mathrm{H}$ & 4.8025 & 4.56852 & 1.2423 \\
\hline 74 & $\mathrm{H}$ & 0.24387 & -7.19806 & 0.13182 \\
\hline
\end{tabular}

\begin{tabular}{|l|l|l|l|l|}
\hline \multicolumn{2}{|c|}{ Conformer D } & \multicolumn{3}{|c|}{$\begin{array}{c}\text { Standard Orientation } \\
\text { (Angstroms })\end{array}$} \\
\hline I & Atom & X & Y & \multicolumn{1}{|c|}{ Z } \\
\hline 1 & C & 2.08692 & 2.04724 & 2.69662 \\
\hline 2 & C & 2.04728 & 3.34429 & 2.2043 \\
\hline 3 & C & 1.11162 & 3.66613 & 1.22624 \\
\hline 4 & C & 0.23024 & 2.69267 & 0.7305 \\
\hline 5 & C & 0.31008 & 1.3683 & 1.18721 \\
\hline 6 & C & 1.22832 & 1.06689 & 2.20673 \\
\hline 7 & C & -0.83437 & 3.06607 & -0.22483 \\
\hline 8 & C & -1.95797 & 2.12948 & -0.46677 \\
\hline 9 & C & -1.83714 & 0.80719 & -0.03284 \\
\hline
\end{tabular}




\begin{tabular}{|c|c|c|c|c|}
\hline 10 & $\mathrm{C}$ & -0.54315 & 0.27222 & 0.56683 \\
\hline 11 & $\mathrm{C}$ & -3.13832 & 2.56576 & -1.10489 \\
\hline 12 & $\mathrm{C}$ & -4.25502 & 1.6989 & -1.30477 \\
\hline 13 & $\mathrm{C}$ & -4.11454 & 0.3666 & -0.83001 \\
\hline 14 & $\mathrm{C}$ & -2.92681 & -0.05597 & -0.21197 \\
\hline 15 & $\mathrm{C}$ & -5.49844 & 2.04615 & -1.9354 \\
\hline 16 & $\mathrm{C}$ & -6.52556 & 1.09192 & -2.06599 \\
\hline 17 & $\mathrm{C}$ & -6.34579 & -0.19394 & -1.58675 \\
\hline 18 & $\mathrm{C}$ & -5.15766 & -0.55788 & -0.97593 \\
\hline 19 & $\mathrm{O}$ & -0.81756 & 4.17262 & -0.76192 \\
\hline 20 & $\mathrm{O}$ & -7.31996 & -1.1407 & -1.69772 \\
\hline 21 & $\mathrm{C}$ & -5.77174 & 3.42545 & -2.48363 \\
\hline 22 & $\mathrm{O}$ & -3.23437 & 3.86591 & -1.54898 \\
\hline 23 & $\mathrm{O}$ & 1.10278 & 4.96819 & 0.80228 \\
\hline 24 & $\mathrm{O}$ & 2.96902 & 1.68124 & 3.66836 \\
\hline 25 & $\mathrm{C}$ & 2.1969 & 1.77974 & -3.49868 \\
\hline 26 & $\mathrm{C}$ & 0.84271 & 1.48972 & -3.59009 \\
\hline 27 & $\mathrm{C}$ & 0.21712 & 0.67821 & -2.64756 \\
\hline 28 & $\mathrm{C}$ & 0.9445 & 0.14637 & -1.56983 \\
\hline 29 & $\mathrm{C}$ & 2.32471 & 0.39194 & -1.5097 \\
\hline 30 & $\mathrm{C}$ & 2.93746 & 1.22235 & -2.46112 \\
\hline 31 & $\mathrm{C}$ & 0.23941 & -0.63894 & -0.47419 \\
\hline 32 & $\mathrm{C}$ & 1.1686 & -1.61433 & 0.23674 \\
\hline 33 & $\mathrm{C}$ & 2.55024 & -1.41442 & 0.28614 \\
\hline 34 & $\mathrm{C}$ & 3.13649 & -0.28671 & -0.47726 \\
\hline 35 & $\mathrm{C}$ & 0.62952 & -2.71757 & 0.91259 \\
\hline 36 & $\mathrm{C}$ & 1.43376 & -3.60313 & 1.64759 \\
\hline 37 & $\mathrm{C}$ & 2.83889 & -3.40861 & 1.73666 \\
\hline 38 & $\mathrm{C}$ & 3.37066 & -2.28525 & 1.03403 \\
\hline 39 & $\mathrm{C}$ & 0.81865 & -4.68065 & 2.29923 \\
\hline 40 & $\mathrm{C}$ & 1.56574 & -5.57705 & 3.04456 \\
\hline 41 & $\mathrm{C}$ & 2.93646 & -5.42508 & 3.16059 \\
\hline 42 & $\mathrm{C}$ & 3.58685 & -4.35403 & 2.5184 \\
\hline 43 & $\mathrm{O}$ & 4.30885 & 0.04244 & -0.30284 \\
\hline 44 & $\mathrm{C}$ & 5.08271 & -4.25731 & 2.69377 \\
\hline 45 & $\mathrm{O}$ & 4.72843 & -2.06212 & 1.09333 \\
\hline 46 & $\mathrm{O}$ & 4.27339 & 1.52251 & -2.44189 \\
\hline 47 & $\mathrm{O}$ & 0.90629 & -6.60258 & 3.65375 \\
\hline 48 & $\mathrm{O}$ & 0.07341 & 1.99192 & -4.59623 \\
\hline 49 & $\mathrm{H}$ & -0.48832 & -1.27943 & -0.9959 \\
\hline 50 & $\mathrm{H}$ & -0.86764 & -0.35763 & 1.40944 \\
\hline 51 & $\mathrm{H}$ & 2.71622 & 4.11426 & 2.57488 \\
\hline
\end{tabular}




\begin{tabular}{|l|l|l|l|l|}
\hline 52 & $\mathrm{H}$ & 1.29563 & 0.06473 & 2.62612 \\
\hline 53 & $\mathrm{H}$ & -2.84802 & -1.08796 & 0.13166 \\
\hline 54 & $\mathrm{H}$ & -7.46042 & 1.36953 & -2.54495 \\
\hline 55 & $\mathrm{H}$ & -5.05638 & -1.57857 & -0.61395 \\
\hline 56 & $\mathrm{H}$ & -8.08846 & -0.74495 & -2.14139 \\
\hline 57 & $\mathrm{H}$ & -5.73244 & 4.17132 & -1.68377 \\
\hline 58 & $\mathrm{H}$ & -5.05843 & 3.67195 & -3.27616 \\
\hline 59 & $\mathrm{H}$ & -6.77143 & 3.49136 & -2.9277 \\
\hline 60 & $\mathrm{H}$ & -2.36423 & 4.30675 & -1.43506 \\
\hline 61 & $\mathrm{H}$ & 0.43617 & 5.04831 & 0.08413 \\
\hline 62 & $\mathrm{H}$ & 3.52926 & 2.44523 & 3.88757 \\
\hline 63 & $\mathrm{H}$ & 2.69279 & 2.41608 & -4.22451 \\
\hline 64 & $\mathrm{H}$ & -0.84642 & 0.4776 & -2.76312 \\
\hline 65 & $\mathrm{H}$ & -0.44663 & -2.8897 & 0.87313 \\
\hline 66 & $\mathrm{H}$ & -0.25605 & -4.83518 & 2.23412 \\
\hline 67 & $\mathrm{H}$ & 3.51562 & -6.13213 & 3.74802 \\
\hline 68 & $\mathrm{H}$ & 5.46711 & -5.06608 & 3.32549 \\
\hline 69 & $\mathrm{H}$ & 5.58906 & -4.34442 & 1.7275 \\
\hline 70 & $\mathrm{H}$ & 5.35162 & -3.31798 & 3.18674 \\
\hline 71 & $\mathrm{H}$ & 4.93069 & -1.20824 & 0.65235 \\
\hline 72 & $\mathrm{H}$ & 4.66488 & 1.11023 & -1.63983 \\
\hline 73 & $\mathrm{H}$ & 1.55295 & -7.14544 & 4.13457 \\
\hline 74 & $\mathrm{H}$ & 0.62331 & 2.5682 & -5.15401 \\
\hline
\end{tabular}

